\title{
1 Viral mediated knockdown of GATA6 in SMA iPSC-derived astrocytes prevents motor neuron loss \\ 2 and microglial activation
}

3 Reilly L. Allison ${ }^{1}$, Emily Welby ${ }^{1}$, Guzal Khayrullina ${ }^{2}$, Barrington G. Burnett ${ }^{2}$, and Allison D. Ebert ${ }^{1}$

$4 \quad{ }^{1}$ Department of Cell Biology, Neurobiology, and Anatomy, Medical College of Wisconsin, Milwaukee, WI

$5 \quad{ }^{2}$ Department of Anatomy, Physiology, and Genetics, Uniformed Services University, Bethesda, MD

6

7 Abstract

8 Spinal muscular atrophy (SMA), a pediatric genetic disorder, is characterized by the profound loss of

9 spinal cord motor neurons and subsequent muscle atrophy and death. Although the mechanisms

10 underlying motor neuron loss are not entirely clear, data from our work and others support the idea that

11 glial cells contribute to disease pathology. GATA6, a transcription factor that we have previously shown

12 to be upregulated in SMA astrocytes, is negatively regulated by SMN and can increase the expression of

13 inflammatory regulator NFkB. In this study, we identified upregulated GATA6 as a contributor to

14 increased activation, pro-inflammatory ligand production, and neurotoxicity in spinal-cord patterned

15 astrocytes differentiated from SMA patient induced pluripotent stem cells. Reducing GATA6 expression

16 in SMA astrocytes via lentiviral infection ameliorated these effects to healthy control levels. Additionally,

17 we found that SMA astrocytes contribute to SMA microglial phagocytosis, which was again decreased by

18 lentiviral-mediated knockdown of GATA6. Together these data identify a role of GATA6 in SMA

19 astrocyte pathology and further highlight glia as important targets of therapeutic intervention in SMA.

\section{Introduction}

22 Spinal muscular atrophy (SMA) is a prevalent genetic cause of infant mortality with an incidence of 1 in 23 every $6,000-10,000$ births. ${ }^{1,2}$ SMA is caused by a loss of function mutation in the survival motor neuron-1

24 (SMN1) gene, which leads to reduced SMN protein expression. ${ }^{3}$ This leads to the selective loss of motor

25 neurons in the spinal cord and the atrophy of skeletal muscle, ultimately resulting in respiratory distress

26 and death. ${ }^{4}$ A second gene, $S M N 2$, produces some functional SMN, but due to alternative splicing this 
27 gene mainly produces a truncated, non-functional protein (SMN $\Delta 7$ ). SMN has been shown to be

28 ubiquitously expressed in all cell and tissue types, though its loss in SMA particularly impacts motor

29 neurons. ${ }^{5}$ SMA motor neurons (MNs) show intrinsic deficits in splicing and electrophysiological function,

30 however restoring SMN to motor neurons alone does not significantly improve disease pathogenesis. ${ }^{6-9}$

31 Currently, there are 3 FDA approved treatments for SMA, two of which focus on correcting or adjusting

32 the splicing of SMN2 (nusinersen, risdiplam), as well as a gene therapy to deliver functional SMN1

33 (onasemnogene abeparvovec). ${ }^{10}$ With recent studies finding potential risk in the AAV9-SMN gene

34 therapy treatment for less severe SMA patients, the clinical trials to expand this treatment have halted. ${ }^{11-13}$

35 The need to identify additional drivers of MN loss remains essential for SMA patient longevity.

37 Glial cells — including astrocytes and microglia —also lose SMN expression in SMA, which results in

38 aberrant activation. Astrocytes are the most abundant cell in the central nervous system (CNS), and

39 microglia are its primary immune cell. In normal physiological function, both have neuroprotective

40 effects and encourage neuronal development, differentiation, and function. Specifically, astrocytes

41 produce and release growth factors like nerve growth factor (NGF), brain derived neurotrophic factor

42 (BDNF), and glial derived neurotrophic factor (GDNF), maintain extracellular concentrations of ions

$43(\mathrm{H}+, \mathrm{K}+)$, free radicals, and neurotransmitters, and provide structural support. ${ }^{14,15}$ Microglia regulate pro-

44 and anti-inflammatory processes and respond to pathological insults through phagocytosis, but also

45 secrete BDNF, provide metabolic intermediates and neurotransmitters, and respond to neuronal firing

46 through the retraction and extension of their highly motile processes. ${ }^{16-18}$ The emerging idea of a quad-

47 partite synapse emphasizes the tightly interdependent and highly responsive nature of communication via

48 secreted factors between astrocytes, microglia, and neurons in the CNS. ${ }^{19}$

50 It is well known that astrocytes and microglia activate in response to stress or injury, and upregulate pro-

51 inflammatory cytokines, downregulate neurotrophic factor secretion, and can induce neuron death. ${ }^{20}$ In

52 disease conditions like SMA, however, the changed interactions between these cells and their contribution 
53 to neuronal loss have not yet been fully characterized. Importantly, astrocyte secreted factors that are

54 known to impact motor neuron health and development, such as glial fibrillary acidic protein (GFAP) and

55 pro-inflammatory cytokines interleukin $1 \beta$ (IL1B) and interleukin 6 (IL6), are also received by microglial 56 membrane receptors. ${ }^{21,22}$ The activity of these canonical receptors, including IL1R1 and IL6R, can serve

57 to start inflammatory mechanisms and transform ramified microglia with small cell bodies and extensive,

58 ramified branches into large, ameboid cells capable of phagocytosis. ${ }^{23-25}$ Complement factors serve as

59 "eat me" tags to activated microglia, and complement proteins $\mathrm{C} 1 \mathrm{q}$ and $\mathrm{C} 3$ have been found to directly

60 contribute to the loss of viable neurons through primary phagocytosis (phagoptosis) in several

61 neurodegenerative diseases. ${ }^{26-29}$ In this way, secreted factors which are essential for astrocytes and

62 microglia to communicate in normal physiological conditions may serve as pathological drivers of

63 activation when dysregulated.

65 Our lab has previously shown that astrocytes in SMA show activation before overt motor neuron loss and

66 can induce detrimental effects on motor neuron cell health and further astrocyte activation. ${ }^{30,31}$ One

67 potential explanation for this effect is the noted change in astrocyte secreted factors, as SMA astrocytes

68 show reduced GDNF release, abnormal microRNA production, and increased NF $\kappa$ B expression. ${ }^{30-34}$

69 GATA6, a zinc finger transcription factor negatively regulated by SMN and associated with NFkB, ${ }^{35,36}$

70 was accordingly found to be increased in SMA mouse and human samples. ${ }^{36}$ Microglial activation has

71 been noted late in the pathology of SMA mouse models, ${ }^{37}$ but recent evidence demonstrates microglial

72 phagocytosis of complement-tagged synapses on proprioceptive neurons in the SMA mouse spinal cord,

73 indicating a role during disease progression. ${ }^{38}$ It is not yet known how upregulated GATA6 changes the

74 astrocyte phenotype in SMA, nor how this impacts astrocyte interactions with motor neurons and

75 microglia. In this study, we utilized spinal-cord patterned astrocytes differentiated from SMA patient and

76 healthy control induced pluripotent stem cells (iPSCs) to investigate the astrocytic role of GATA6 in

77 aberrant activation and neurotoxicity as well as astrocyte-driven microglia activation in vitro. We

78 observed an increase in pro-inflammatory factor production in astrocytes associated with increased 
GATA6 expression. Lentiviral mediated GATA6 overexpression was found to increase motor neuron cell loss in vulnerable SMA cultures, whereas lentiviral mediated knockdown ameliorated this effect. SMA astrocytes were also found to increase activation and phagocytic ability of SMA microglia, and again GATA6 astrocyte knockdown ameliorated these effects. Together these data indicate a role for GATA6 as a major contributor to the aberrant activation and pathological functions of glial cells in SMA.

\section{Results}

\section{Differentiation of spinal cord astrocytes from induced pluripotent stem cells}

87 Though astrocytes in general share a common goal of supporting neuronal function, subtle differences in

88 gene expression, morphology, and function in astrocytes between regions of the brain and spinal cord

89 have been identified. ${ }^{39,40}$ To best model astrocyte subtypes that would interact most directly with

90 degenerating motor neurons in SMA, we differentiated ventral-caudal patterned neural progenitor cells

91 (NPCs) into spinal cord-like astrocytes. We utilized several recently updated astrocyte protocols to

92 optimize our own culture conditions. ${ }^{41-44}$

Human induced pluripotent stem cells (iPSCs) from SMA patients and healthy (unaffected)

94 individuals, hereafter referred to as "SMA" and "Ctrl," were differentiated into neural progenitor cells

95 (NPCs) through the addition of SMAD inhibitors (SMADi) with retinoic acid (RA) and smoothened

96 agonist (SAG) to mimic spinal cord development (Figure 1A). Plating NPCs at a high density was

97 necessary to achieve robust astrocyte differentiation (Figure 1B; NPC P2 Day 17). These NPCs were

98 found to express known NPC-associated genes such as NESTIN, SOX2, and PAX6 (Figure 1C). They

99 also expressed spinal cord ventral-caudal associated genes including ISL1, NKX6.1, OLIG2, HOXB4,

100 and HOXA4, which showed similar gene expression trends to post-mortem spinal cord from SMA

101 patients and unaffected individuals (Figure 1C). These spinal cord genes were not upregulated in SMADi

102 only treated NPCs, which instead showed increased expression of forebrain associated genes (TBR2,

103 SIX3, OTX2, Figure 1C). 

specific medium. A small neuronal population was noted during early stages of astrocyte differentiation

Ventral-caudal patterned astrocytes were found to express characteristic markers of astrocyte identity and function such as S100ß (Figure 1D), VIMENTIN (Figure 1E), ALDH1L1, APOE, and GLUL (Figure 1F), which showed similar expression trends to human spinal cord samples (Figure 1G). iPSC-derived astrocytes and human spinal cord samples also showed equivalent expression of the spinal cord ventral-

111 caudal transcription factor HOXB4 (Figure 1F, G). The Ctrl and SMA astrocytes were found to secrete

112 interleukin 6 (IL6) into the media, with SMA astrocytes secreting slightly higher levels of IL6 at baseline

$113(\mathrm{p}=0.0769, \mathrm{~ns}$, Figure 1H). When stimulated with polyinosinic-polycytidylic acid (poly:IC), a compound

114 that activates inflammatory responses via toll-like receptors, ${ }^{45} \mathrm{Ctrl}$ astrocytes slightly increased IL6

115 secretion though not significantly, whereas SMA astrocytes significantly upregulated IL6 secretion 116 compared to untreated and PBS-treated SMA astrocyte samples $(\mathrm{p}<0.005$, Figure $1 \mathrm{H})$. These data fit with 117 the findings from our previous model ${ }^{30,32}$ and establish these spinal cord astrocytes as phenotypically and 118 functionally consistent with other in vitro models. ${ }^{41,42,46,47}$

121 Our lab previously reported decreased GDNF production and increased production of GATA6 in SMA

122 astrocytes compared to healthy controls. ${ }^{32}$ These findings are consistent with our updated differentiation

123 in which RNA transcripts of GDNF and BDNF were both significantly downregulated in the SMA

124 astrocytes compared to Ctrl (Figure $2 \mathrm{~A}, \mathrm{p}=0.0033,0.0221$ ). GATA6 and NFkB transcripts were found to

125 be significantly upregulated in the SMA cultures, also confirming our previous findings (Figure 2B,

$126 \mathrm{p}<0.0001, \mathrm{p}=0.0001)$. These transcript changes were maintained in protein expression as confirmed by

127 immunocytochemistry (ICC) $(\mathrm{p}=0.0187$, Figure 2C-E).

128 Importantly, phosphorylated $\mathrm{NF \kappa B}(\mathrm{phNF \kappa B})$ was also found to be upregulated in the nuclei of

129 SMA astrocytes compared to Ctrl (Figure 2D-F, IHC p=0.0031). phNFאB is known to enter the nucleus 
and regulate the gene expression of pro-inflammatory cytokines. ${ }^{48,49}$ Accordingly, we found upregulated transcripts for interleukin 1 beta (IL1 $)$ ) and interleukin 6 (IL6), as well as upregulated complement cascade members C1q and C3 in the SMA astrocyte cultures (Figure 2G, p=0.0741, 0.0006, 0.0198, 0.0009). Glial fibrillary acidic protein (GFAP), a canonical marker of astrocyte activation, was also found upregulated, though none of these changes were associated with any changes in the general astrocyte marker S100B expression (Figure 2H, $\mathrm{p}<0.0001,0.8529$ ). Overall, these findings confirm the increased activation of SMA astrocytes compared to healthy control astrocytes in our updated culture system. They also identify GATA6 and NFkB as potential drivers of increased inflammatory ligand production in

\section{SMA.}

\section{Manipulation of GATA6 associated with changes in reactivity and neurotrophic support}

141 To directly examine the influence of GATA6 upregulation on driving astrocyte activation, we infected healthy control astrocytes (Ctrl) with a lentivirus expressing GATA6 (GATA6OE) or a GFP control. We also separately infected SMA astrocytes with a lentivirus expressing an shRNA against GATA6

144 (GATA6KD) construct or GFP control. We first confirmed GATA6 viral expression or knockdown in 145 RNA transcripts by qRT-PCR (Figure 3A) and confirmed its protein expression by ICC (OE fold change 146 of 9.7576, KD fold change of -3.2181, Figure 3C, E). Ctrl GATA6OE astrocytes showed increased 147 GATA6 levels equivalent to SMA astrocyte levels (Ctrl GATA6OE to SMA GFP qPCR: p=0.9665, ICC $148 \mathrm{p}=0.2320$ ), and SMA GATA6KD astrocytes were found to have decreased GATA6 levels similar to Ctrl 149 astrocyte baseline (SMA GATA6KD to Ctrl GFP qPCR:0.0630, ICC p=0.9783). Interestingly, no

150 significant changes in NFKB transcript levels were observed in Ctrl GATA6OE samples compared to Ctrl 151 GFP samples (Figure 3B), although nuclear phNFkB protein levels were found significantly increased

152 (ICC $\mathrm{p}=0.0178$, Figure 3F). GATA6KD in the SMA astrocytes did have an associated significant

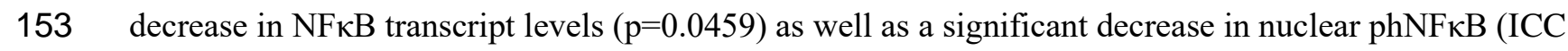
$154 \mathrm{p}=0.0289$, Figure 3B, 3D, 3F). 
In relation to the NFKB data, we examined canonical pro-inflammatory cytokines in astrocyte samples, finding a significant increase in secreted IL6 after GATA6OE (Ctrl GFP to Ctrl GATA6OE p<0.0001, Figure 3G) and a significant decrease after GATA6KD (SMA GFP to SMA GATA6KD p=0.0002, Figure 3G). No significant changes in IL1B were detected (Figure 3H). Significant decreases in C1q ( $p=0.0004)$ and C3 ( $\mathrm{p}=0.0035)$ transcript levels were also found after GATA6KD, and there was only a slight increase in expression after GATA6OE (fold change $=0.4286,0.9898$, ns) (Figure 3H). No significant changes in neurotrophic factors or astrocyte marker S100B were found in any condition (Figure 3I, 3J).

GFAP transcript was significantly reduced after GATA6KD in SMA astrocytes $(\mathrm{p}=0.0087)$ but was not significantly increased in GATA6OE Ctrl astrocytes (fold change $=0.6500$, ns, Figure 3J). These findings suggest that aberrant GATA6 expression in SMA astrocytes is sufficient to modulate inflammatory ligands, complement components, and GFAP transcripts.

\section{Effect of astrocyte GATA6 manipulation on spinal motor neuron survival}

We next investigated whether GATA6-associated reactivity and inflammation in astrocytes contributed to spinal motor neuron loss in vitro. Following the established Maury et al. protocol ${ }^{50}$ for generating spinal motor neurons (MNs) from human iPSCs, we matured plated MNs for 21-28 days before analysis. To

171 examine the possible contribution of GATA6 to SMA astrocyte neurotoxicity, conditioned media (ACM)

172 from Ctrl, Ctrl-GFP, Ctrl-GATA6OE astrocytes, as well as SMA, SMA-GFP, and SMA-GATA6KD

173 astrocytes was applied onto Ctrl and SMA MNs. Cell death was then measured using a TUNEL assay and 174 normalized to a live cell counterstain (7AAD, Figure 4A\&B, D\&E). Quantification of percent change was 175 calculated using normalized values compared to one Ctrl UTX ACM replicate for Ctrl and SMA MNs 176 (Figure 4C, F). Consistent with our previous findings, in both Ctrl and SMA MNs, ACM from untreated SMA

178 astrocytes induced more cell death than ACM from untreated Ctrl (Ctrl UTX) astrocytes ( $\mathrm{p}=0.0181$,

179 0.0354, Figure 4A-4C, 4D-4F). No significant differences were found between the untreated and GFP180 control samples in either Ctrl or SMA astrocytes (Ctrl- Ctrl UTX:GFP p=0.9992, Ctrl-SMA UTX:GFP 

significantly increase cell death compared to Ctrl UTX ACM in SMA MNs ( $p=0.0001)$, though not in Ctrl MNs ( $\mathrm{p}=0.9850)$. This difference is likely due to the inherent vulnerability of SMA MNs. Strikingly, SMA GATA6KD ACM significantly decreased MN cell death in both Ctrl and SMA MNs compared to SMA UTX ACM $(p=0.0021,0.0128)$. These data support the idea that GATA6 overexpression in SMA astrocytes drives neurotoxicity.

\section{Effect of astrocyte activation and GATA6 manipulation on microglial activation}

189 Finally, we examined the effect of SMA astrocyte activation on human iPSC-derived microglia from

190 SMA patients and healthy controls. Many models of neurodegeneration establish a role for activated

191 microglia in driving further neuron loss..$^{27,51-56}$ Because many of the increased pro-inflammatory cytokines

192 and complement components found within our SMA astrocyte cultures are key signals for microglial

193 activation and phagocytosis, we aimed to examine the possibility of SMA astrocyte-driven microglial

194 activation contributing to spinal motor neuron loss. We started by generating microglia from SMA and

195 Ctrl iPSC lines using established differentiation protocols and commercial kits (Figure 5A). ${ }^{57,58}$ Generated

196 microglia were confirmed TMEM119+, P2RY12+, and Iba1+ before analyses (Figure 5B). microglia (Figure 5B). Though no differences were significant, we did find trends for increased pro-

199 inflammatory receptor transcripts including C3aR1, CXCR1, CSFRA, IL1R1, and IL6R in SMA

200 microglia (Figure 5B). To test if SMA microglia may have heightened sensitivity to astrocyte secreted

201 factors, we first applied Ctrl UTX and SMA UTX ACM onto SMA microglia generated from a yolk-sac

202 lineage protocol (Figure 5C, 5D). Ctrl ACM increased microglia mobility - a sign of non-activated,

203 surveilling microglia ${ }^{18,59}$ - compared to control media treated cells as measured by distance traveled

204 through a live-image tracking assay ( $\mathrm{p}=0.0316$, Figure 5C; image). The application of SMA ACM onto

205 SMA microglia significantly reduced this surveilling phenotype ( $\mathrm{p}=0.0009$, Figure $5 \mathrm{C})$. To more directly

206 examine functional differences, we measured phagocytic ability using fluorescent bead-based flow 
cytometry (Figure 5D). We found a significantly increased number of phagocytic SMA microglia after treatment with SMA ACM than Ctrl ACM (p=0.0350, Figure 5D).

We then wanted to examine the role of GATA6 in the SMA astrocyte-driven microglial activation

210 phenotype and confirm our findings in a hematopoietic lineage model of microglia (Figure 5E, 5F). First,

211 we found no differences in soma diameter - a sign of activation ${ }^{60}$ - between Ctrl UTX, Ctrl GATA6OE,

212 or SMA UTX ACM applied onto Ctrl microglia ( $\mathrm{p}=0.3193,0.6850$, ns, Figure 5E). A slight but

213 significant decrease in diameter was seen in the SMA GATA6KD treated Ctrl microglia compared to Ctrl

214 GATA6OE ( $\mathrm{p}=0.0154$, Figure 5E). In the SMA microglia treatments, a significant increase in size was

215 seen in the Ctrl GATA6OE condition, comparable to SMA UTX treated microglia (Ctrl UTX:GATA6OE

$216 p<0.0001$, GATA6OE:SMA UTX p=0.7106, Figure 5E). SMA microglia treated with SMA GATA6KD

217 ACM showed a significant decrease in diameter, reducing sizes to Ctrl UTX levels (SMA

218 UTX:GATA6KD p=0.0422, Ctrl UTX: GATA6KD p=0.9986). No differences were found between Ctrl

219 UTX and Ctrl GFP, or SMA UTX and SMA GFP conditions ( $\mathrm{p}=0.1295,0.8410)$. Functionally, a

220 significant decrease in phagocytic cells was found in SMA microglia treated with the SMA GATA6KD

221 ACM compared to SMA UTX or SMA GFP ACM ( $p=0.0102,0.0019$, Figure 5F). A significant increase

222 in phagocytic cells was also found in after treatment with the Ctrl GATA6OE ACM compared to Ctrl

$223 \operatorname{UTX}$ ACM $(p=0.0014$, Figure 5F).

Finally, we aimed to test if the activating or toxic effect was unique to SMA astrocytes or if SMA microglia had similar activating effects. Therefore, we treated SMA and control astrocytes with SMA or control microglia conditioned medium (MCM). Interestingly, no differences in activation were found

227 when astrocytes were treated with media conditioned by Ctrl or SMA microglia (ns, Figure 5G). GATA6

228 transcripts were not differentially expressed between SMA and Ctrl microglia or between SMA and Ctrl

229 motor neurons analyzed by RNAseq (data not shown) suggesting aberrant GATA6 expression is more

230 astrocyte restricted. Overall, these data suggest a role for SMA astrocytes in SMA microglial activation

231 and supports a role of aberrant GATA6 expression as a driving factor in astrocyte mediated neurotoxicity

232 and microglial phagocytosis. 


\section{Discussion}

235 The loss of SMN in SMA causes selective lower motor neuron loss in patients, and this is replicated in

236 vivo with SMA mouse models. ${ }^{61}$ However, in purified motor neuron cultures in vitro, SMA iPSC-derived

237 motor neurons do not show increased cell death compared to healthy controls. ${ }^{32,62}$ This recent distinction,

238 along with a body of evidence showing that targeting SMN restoration to only motor neurons has limited

239 effect, suggest a role for non-cell autonomous contributions to SMA motor neuron vulnerability..$^{6,7,9,63}$ In

240 parallel, pre-synaptic deficits and aberrant activation of glial cells in SMA have been observed before

241 overt motor neuron loss. ${ }^{30}$ Specifically, SMA astrocytes have been shown to decrease neurotrophic and

242 growth factor production and increase the pro-inflammatory production of GFAP, IL6, and IL1B. ${ }^{30,32,34}$

243 The application of SMA astrocyte conditioned media has been shown to have detrimental effects on both

244 healthy control MNs and those with reduced SMN. ${ }^{32,64}$ Activated (Iba1+, M1) microglia have also been

245 found to be increased at early symptomatic stages and localized to motor neurons in SMA rodents. ${ }^{37,65,66}$

246 Synapse loss has been associated with microglial phagocytosis, with complement factors C1q and C3

247 implicated in targeting microglia removal of healthy synapses. ${ }^{38,66}$ Together, these data robustly indicate a

248 role for glia in contributing to motor neuron loss in SMA.

250 Here, we utilized human iPSC-derived astrocytes, microglia, and spinal motor neurons from healthy

251 control and SMA patient lines to examine disease properties in human cells. Though all astrocytes are

252 thought to share common features (e.g., upregulation of GFAP after activation) and functions (e.g.,

253 formation and maintenance of the blood-brain barrier), recent studies have identified differences in gene

254 expression, morphology, and function between astrocytes in distinct regions of the CNS. ${ }^{39,67}$ Even within

255 the spinal cord, three distinct populations of astrocytes are hypothesized to preferentially support neurons

256 from the same progenitor subtypes..$^{40,68,69}$ To address these emerging ideas, we started by developing a

257 more disease-relevant astrocyte culture by patterning neural progenitor cells with small molecules to

258 mimic spinal cord development, then differentiated these into functional spinal cord astrocytes. ${ }^{39,40,70} \mathrm{We}$ 
found that SMA astrocytes upregulated classical markers of activation, decreased neurotrophic support, and increased pro-inflammatory ligands and complement components compared to controls. These data establish our spinal cord astrocytes as a disease-relevant and functional model concurrent with others being used in the field..$^{41,42,46,47}$ They also allow us to examine astrocyte activation and interactions with spinal motor neurons and microglia in an SMA-specific context throughout the rest of this study.

In order to more specifically investigate the proposed relationship between SMN, GATA6, and NFkB in the context of astrocyte activation, we infected astrocytes with a lentivirus to drive GATA6 expression in Ctrl astrocytes or knock it down in SMA astrocytes. Interestingly, we found that though the transcript and protein levels of GATA6 were successfully manipulated, only the knockdown of GATA6 in SMA astrocytes, not the overexpression in $\mathrm{Ctrl}$ astrocytes, was associated with a significant change in NFkB transcript levels. Both, however, were associated with increased nuclear phNFkB protein levels.

271 Knocking down GATA6 in the SMA astrocytes significantly decreased inflammatory and activation 272 marker transcripts, though it did not restore neurotrophic support through BDNF or GDNF. Within these 273 SMN-deficient SMA astrocytes, GATA6 does appear to be a main contributor of aberrant activation, 274 confirming what transcriptome analyses, in vivo studies, and our own previous findings predicted. ${ }^{32,36}$ In 275 contrast, overexpressing GATA6 in the Ctrl astrocytes showed trending — but not significant—increases 276 in these transcripts. This suggests that these inflammatory ligands are more tightly regulated by NFkB

277 than GATA6, with GATA6 potentially competing with functional alternative regulators of NFkB in 278 healthy SMN-expressing astrocytes. ${ }^{71}$ The relationship between GDNF, BDNF, and GFAP may be 279 similarly complicated. Histone deacetylase (HDAC), an enzyme which can increase DNA susceptibility 280 to transcription factor (TF) regulation, has shown inverse associations with GFAP compared to BDNF 281 and GDNF. In different contexts, low HDAC (DNA more accessible for TF regulation) is associated with 282 decreased GFAP, whereas high HDAC (DNA less accessible for regulation) is associated with increased 283 BDNF and GDNF. ${ }^{72,73}$ These complicated associations with the disease-specific genetic changes may 
284 begin to illuminate why the impact of GATA6KD was more striking in the SMA astrocytes than its overexpression in controls. pattern. Expectedly, SMA ACM was found to increase motor neuron cell death in SMA and Ctrl cultures compared to Ctrl ACM, and the knockdown of GATA6 in SMA astrocytes ameliorated this effect in both SMA and Ctrl MNs. Strikingly, the overexpression of GATA6 in Ctrl astrocytes increased cell death only in the SMA MNs, not in the healthy controls. This again could be due to regulatory mechanisms-like those involving SMN-which are intact in the Ctrl MNs but are absent in SMA MNs leaving them more vulnerable to even slight changes in astrocyte secreted factors. One example of this is the SMNdeficiency-linked activation of p53 found in vulnerable motor neurons at pre-symptomatic stages in SMA mice. ${ }^{32}$ This distinction between GATA6OE toxicity to Ctrl and SMA MNs emphasizes the importance of therapeutically targeting the early-activating SMA astrocytes, especially in patients not receiving the AAV therapy to restore SMN. It also provides an interesting topic of future study regarding the resistance of AAV9-SMN treated motor neurons to astrocyte toxicity, specifically regarding GATA6-driven toxicity. differentiating microglia from SMA and healthy control iPSCs. At baseline, we observed differences between the Ctrl and SMA microglia in pro-inflammatory receptors known to activate microglia from a neurotrophic, surveilling phenotype — characterized by small soma and highly motile processes — towards an ameboid, phagocytic phenotype..$^{23,24,59} \mathrm{We}$ also identified upregulated complement receptor C3aR1, a

306 receptor which has been specifically tied to microglial activation and phagocytosis of neurons or synapses 307 in several models of neurodegeneration..$^{27,74-76}$ The upregulation of these receptors in SMA microglia may 308 prime them for the upregulated ligands produced by SMA astrocytes to encourage activation.

309 Encouragingly, we found that SMA ACM applied onto SMA microglia increased activated phenotypes 
compared to Ctrl ACM or no treatment in both yolk-sac lineage and hematopoietic progenitor lineage

311 microglia. These data first confirmed the ability of iPSC-derived astrocytes and microglia to communicate

312 via secreted factors. Additionally, they suggest that microglia-mediated phagocytosis could contribute to

313 viable neuron loss in SMA, as has been suggested in neurodegenerative models such as Alzheimer's and

314 Parkinson's disease. ${ }^{77-79}$ Importantly, the use of individually differentiated cultures and ACM treatments

315 in this study suggest that microglia activation in SMA may be fueled by activated astrocyte secreted

316 factors.

318 Finally, we examined the potential impact of GATA6 knockdown on this astrocyte-driven microglial

319 activation. Morphologically and functionally, we found that treatment with SMA UTX and Ctrl

320 GATA6OE ACM increased activated phenotypes of SMA microglia compared to Ctrl UTX or SMA

321 GATA6KD ACM. The role of the complement proteins - especially C3 - within this process is one

322 promising area for future studies. C3 is upstream of complement cascade products which can be secreted

323 to act as tags for phagocytosis (C3b, C5a), activators for immune cells (C3a), or initiators of the

324 membrane attack complex (C5b) ${ }^{80}$ In SMA, already vulnerable MNs receiving neurotoxic conditioning

325 and C5b signals from activated astrocytes may be upregulating their own $\mathrm{C} 3$ in addition to initiating the

326 terminal pathway towards cell lysis. ${ }^{81,82}$ Excess complement products then may be additionally tagging

327 nearby healthy neurons for phagocytosis-mediated cell death (i.e. phagoptosis) as well as functional

328 synapses for removal by astrocyte-activated microglia. More experiments to examine the production of

329 C3 by SMA MNs before and after ACM treatment, as well as in MN-microglia co-cultures, are needed to

330 investigate this hypothesis. Nevertheless, these data demonstrate the ability of iPSC-derived astrocytes

331 and microglia to communicate via secreted factors and provide compelling evidence for astrocyte-driven

332 activation of microglia in SMA. Of particular interest, this activation appears to be an astrocyte-first

333 mechanism, as the application of microglia conditioned media onto SMA astrocytes showed no effect

334 This is in contrast to microglia-first activation theories which have been proposed in the context of

335 Alzheimer's, Huntington's, and Parkinson's disease. ${ }^{83-85}$ This is an area of future research, as C3- 
336 expressing activated astrocytes were observed in post-mortem tissue from patients with Alzheimer's,

337 Huntington's, and Parkinson's disease, as well as multiple sclerosis (MS) and amyotrophic lateral

338 sclerosis (ALS). ${ }^{86}$ It would be especially of interest to compare the activation timelines of SMA and ALS,

339 as motor neurons are particularly affected in these disorders - despite quite dissimilar genetic causes-

340 and microglia have been directly linked to motor neuron loss in ALS. ${ }^{87,88}$ Using our isolated iPSC-derived

341 culture system and treatments of ACM or MCM, we may be able to parse out if this astrocyte-first

342 activation is specific to SMA, or if in vivo and post-mortem studies are missing early mechanisms of

343 neuron loss..$^{89}$

345 Together, these data provide evidence to support the predicted role of GATA6 in astrocyte activation and

346 its neurotoxic effects on spinal motor neurons in SMA. They also suggest a role for astrocyte-driven

347 microglia activation, which may contribute to MN death within SMA. More consideration for astrocytes

348 and microglia when designing therapeutics for SMA may positively impact MN health to improve

349 lifespan and function for patients.

$351 \quad$ Materials and Methods

\section{Pluripotent Stem Cells}

353 Two healthy control iPSC lines $(21.8,4.2)$ and three SMA patient iPSC $(7.12,3.6,8.3)$ lines were utilized

354 in these experiments. ${ }^{32}$ All pluripotent stem cells were maintained on Matrigel (Corning) in Essential 8

355 (Gibco) and passaged every 4-6 days. The iPSCs and differentiated cells were confirmed mycoplasma

356 negative.

357 Astrocyte differentiation

358 Spinal cord patterned astrocytes were generated from iPSC-derived neural progenitor cells (NPCs).

359 Catalog numbers for each reagent are listed in supplemental materials (Table S1) and a protocol 
schematic is shown in Figure 1A. Briefly, iPSCs were grown to confluency, dissociated with Accutase, and plated at 2 million cells/well into Matrigel (Corning) coated 6 well-plates in NPC base medium (50\% DMEM/ F12, 50\% Neurobasal, 2\% B27, 1\% N2, 1\% Antibiotic/ Antimycotic, 0.1\% B-mercaptoethanol, 50ng/mL Laminin, and 0.5uM Ascorbic Acid) supplemented with 10uM Y-27632, 3uM Chir-99021, 40uM SB431542, and 0.2uM LDN193189. On day 1, NPC media was changed and supplemented with 3uM Chir-99021, 40uM SB431542, and 0.2uM LDN193189. Cells were washed with PBS if a lot of cell death was observed. Days 2 and 3 involved NPC media changes supplemented with 3uM Chir-99021, 40uM SB431542, 0.2uM LDN193189, 100nM retinoic acid (RA), and 500nM hedgehog smoothened agonist (SAG). From day 4 onwards, NPC media was supplemented with 40uM SB431542, 0.2uM LDN193189, 100nM RA, and 500nM SAG and changed daily. Cells were passaged via Accutase treatment on Day 6 (P1), Day 12 (P2), and Day 18 (P3). Plating density for NPC P1 and P2 is 7 million

371 cells per well (of a 6 well plate) and plating density for NPC P3 to begin astrocyte differentiation (P0) is 372150,000 cells/well (of a 6 well plate).

373 Astrocyte differentiations were cultured with ScienCell Astrocyte Medium containing 1\% Astrocyte

374 Growth Supplement, 1\% Penicillin/Streptomycin, and 2\% B27. Cells were fed every 48 hours and 375 passaged with Accutase every 6-9 days upon confluency (minimum of 3 passages). Astrocyte P1, P2, and 376 P3 were all plated at 150,000 cells/ well, at P4 cells were considered differentiated and split onto

377 coverslips for IHC, into 6 well plates for RNA or protein, or into T75 tissue culture treated flasks for

378 ACM generation and collection. Astrocyte conditioned media was collected upon each media change after

$379 \mathrm{P} 4$, spun to remove any cells or debris, and stored in sterile Falcon tubes at -20C. Frozen medias were

380 slowly thawed on ice before use. Poly:IC treatments were diluted to $100 \mathrm{ng} / \mathrm{mL}$ in supplemented Astrocyte 381 media and left for 24 hours. Cells were then rinsed with PBS and fed with supplemented Astrocyte media.

382 Media was collected from stimulated astrocytes after 48 hours, and pellets were collected for analyses.

383 IL-6 ELISA assay was performed on collected media (Affymetrix eBioscience, \#88-7066-22).

\section{Motor neuron differentiation and treatments}


Spinal motor neurons were differentiated based on the Maury et al. (2015) protocol..$^{50}$ Briefly, embryoid bodies were generated from iPSCs and patterned in the presence of Chir-99021 with dual SMAD inhibition (SB 431542 and LDN 1931899) followed by treatment with retinoic acid (RA), smoothened agonist (SAG), and DAPT. Spinal motor neuron progenitor cells were then dissociated and plated on Matrigel-coated glass coverslips for terminal differentiation and maturation in growth factor supplemented medium for 28-42 days in vitro. Treatments with ACM were performed with $20 \%$ ACM in MN Maturation media after day 28 and left for 48 hours at 37C before fixing, collection, or analyses.

\section{Microglia differentiations}

Microglia were differentiated using the protocols established by Haenseler et al. $(2017)^{57}$ or the newly available differentiation kit by STEMCELL Technologies based on the McQuade et al. (2018) protocol ${ }^{58}$ (\#05310, \#100-0019, \#100-0020). Briefly, iPSCs were patterned into macrophages (pMacs) using BMP4, VEGF, SCF, IL3, and M-CSF. pMacs were matured into microglia (pMGL) using N2, IL34, and GM-

397 CSF (experiments shown in Figures 5C, 5D). Alternatively, iPSCs were differentiated into hematopoietic 398 progenitor cells (HPCs) using the STEMdiff Hematopoietic Kit (StemCell Technologies). Floating HPCs 399 were then collected and plated at 50,000 cells/mL in STEMdiff Microglia Differentiation media

400 (StemCell Technologies) for 24 days, followed by rapid maturation in STEMdiff Microglia Maturation 401 media (StemCell Technologies) for at least 4 days before usage (experiments shown in 5B, 5E, 5F, 5G).

402 Microglia were confirmed TMEM119+, CD45+, and CD11b+ (Figure 5B).

404 RNA was isolated from control and SMA cell pellets using the RNeasy Mini Kit (Qiagen) following 405 manufacturer's instructions, quantified using a Nanodrop Spectrophotometer, treated with RQ1 Rnase-

406 free Dnase (Promega), and converted to cDNA using the Promega Reverse Transcription system

407 (Promega). SYBR green qRT-PCR was performed in triplicate using cDNA and run on Bio-Rad CFX384

408 real time thermocycler. Primers for each target are available in Supplemental materials (Table S2). Cq 
values for each target gene were normalized to housekeeping gene (GAPDH) values and Ct values for

410 each sample were normalized to a human cortex sample to calculate relative fold change (ddCT) in gene

411 expression.

\section{Immunocytochemistry}

413 Plated cells were fixed in 4\% paraformaldehyde (PFA) for 20 minutes at room temperature and rinsed

414 with PBS. Nonspecific labeling was blocked and the cells permeabilized with $0.25 \%$ Triton X-100 in PBS

415 with $1 \%$ BSA and $0.1 \%$ Tween 20 for 15 minutes at room temperature. Cells were incubated with

416 primary antibodies overnight at $4 \mathrm{C}$, then labeled with appropriate fluorescently-tagged secondary

417 antibodies. Hoechst nuclear dye was used to label nuclei. Primary antibodies used were chicken anti-

418 Vimentin (abcam, ab24525), mouse anti-S100ß (Sigma, S2532), rabbit anti-GATA6 (Cell Signaling

419 \#5851), mouse anti-NFkB p65 (Cell Signaling \#6956), and rabbit anti-phospho-NFkB p65 (Cell Signaling

420 \#3033). Secondary antibodies used were donkey anti-mouse AF488 (Invitrogen), goat anti-chicken

421 AF568 (Invitrogen), donkey anti-rabbit AF546 (Invitrogen), and donkey anti-mouse AF647 (Invitrogen).

422 All primaries were used at a 1:500 dilution, and all secondaries were used at 1:1000 dilution. Analyses

423 were performed on three randomly selected fields per coverslip using standard fluorescent microscopy

424 using equivalent exposure conditions. Total fluorescence in each channel was measured using FIJI

425 (ImageJ) software.

TUNEL assay

427 Plated cells were fixed in 4\% PFA for 20 minutes at room temperature, rinsed with PBS, and then stained 428 using a TUNEL Assay Kit - BrdU-Red (abcam, ab66110). Manufacturer's instructions were adapted for

429 coverslips instead of Coplin jars. Briefly, cells were permeabilized as described for ICC, washed, and

430 incubated with DNA labeling solution for 1 hour at 37C. Cells were washed and optional 7AAD nuclear

431 counterstain was then applied for 30 minutes at room temperature. Coverslips were imaged within 3 hours

432 of staining with standard fluorescent microscopy. Three images were acquired from randomly selected 
433 fields for each coverslip. Images were analyzed for total fluorescence in either channel using FIJI

434 (ImageJ) software. Representative images were acquired on a Zeiss confocal microscope within 3 hours

435 of staining.

436 Microglia assays

437 Microglia were treated with 1:2 astrocyte conditioned media with microglia maturation media for 24

438 hours at 37C. Live image tracking was performed with 1:6000 Invitrogen Cell Tracker Green CMFDA

439 dye with 5,000 cells per condition. Images were acquired over 16 hours using an Olympus IX83 Inverted

440 Light Microscope and analyzed on ImageJ using OlympusViewer, TrackMate, and Chemotaxis Tool

441 plugins. Soma size measures were generated by fixing cells onto glass coverslips and staining with Iba1

442 (abcam, ab5076), then acquiring three random images per coverslip. Images were blindly analyzed using

443 Nikon Elements Advanced Research package to measure soma diameter of total $n=50$ cells per condition.

444 Phagocytosis assay was performed by treating cells with astrocyte conditioned media or microglia

445 maturation media (control) for 24 hours at 37C. Cells were then incubated with FluoSpheres Sulfate

446 Microspheres (ThermoFisher \#F8851) for 15 minutes at 37C. Unbound beads were removed from cell

447 suspension, then cells and bound beads were suspended in FACS flow buffer on ice. Flow cytometry was

448 performed immediately and gated on live cells to remove any unbound beads from downstream analyses.

449 Flow cytometry was performed on an LSRFortessa X-20 Special Order Research Product or BD Accuri

450 C6 (BD Biosciences, Franklin Lakes, NJ).

\section{$451 \quad$ Statistical analyses}

452 Experimental conditions within each experiment were performed in technical triplicates for a minimum of

453 three independent experiments. Data were analyzed using GraphPad Prism software and the appropriate

454 statistical tests including the Student's t-test, 1-way ANOVA, and 2-way ANOVA followed by Tukey's

455 post hoc analysis of significance. Changes were considered statistically significant when $\mathrm{p}<0.05$.

\section{Acknowledgements}


457 We would like to acknowledge the Children's Research Institute FLOW Core at the Medical College of

458 Wisconsin and thank Melissa Whyte for her help with the microglia flow cytometry assays. GATA6

459 knockdown and overexpression constructs were generously provided by Dr. Stephen Duncan, and

460 lentiviruses were generated in the Blood Research Institute/ MCW Viral Vector Core. We thank Dr.

461 Michele Battle for helpful GATA6 discussions. This work was supported by grants to A.D.E. from the

462 National Institutes of Health (R21NS102911) and Cure SMA. B.G.B is supported by the NIH

463 (R01NS119594).

464 Author Contributions

465 R.L.A, E.W., and G. K. performed and analyzed experiments. R.L.A, E.W., A.D.E., G.K., and B.G.B

466 designed experiments and interpreted data. A.D.E. and B.G.B supervised the study and provided funding.

467 R.L.A wrote the manuscript, E.W. and R.L.A created figures and legends, and E.W. and A.D.E. edited the 468 manuscript.

\section{Declarations of Interests}

470 The authors declare no competing interests. 


\section{References}

478

479

480

481

482

483

484

485

486

487

488

489

490

491

492

493

494

495

496

497

498

499

500

501

502

503

504

505

506

507

508

509

510

511

512

513

514

515

516

517

518

519

520

521

522

523

524

525

526

1. Oskoui M, Kaufmann P. Spinal muscular atrophy. Neurotherapeutics. Oct 2008;5(4):499506. doi:10.1016/j.nurt.2008.08.007

2. D'Amico A, Mercuri E, Tiziano FD, Bertini E. Spinal muscular atrophy. Orphanet J Rare Dis. Nov 02 2011;6:71. doi:10.1186/1750-1172-6-71

3. Sumner CJ, Paushkin S, Ko C-P. Spinal Muscular Atrophy: Disease Mechanisms and Therapy. 2016.

4. Lefebvre S, Bürglen L, Reboullet $S$, et al. Identification and characterization of a spinal muscular atrophy-determining gene. Cell. Jan 1995;80(1):155-65. doi:10.1016/00928674(95)90460-3

5. Chaytow H, Huang YT, Gillingwater TH, Faller KME. The role of survival motor neuron protein (SMN) in protein homeostasis. Cell Mol Life Sci. Nov 2018;75(21):3877-3894. doi:10.1007/s00018-018-2849-1

6. Gogliotti RG, Quinlan KA, Barlow CB, Heier CR, Heckman CJ, Didonato CJ. Motor neuron rescue in spinal muscular atrophy mice demonstrates that sensory-motor defects are a consequence, not a cause, of motor neuron dysfunction. J Neurosci. Mar 2012;32(11):3818-29. doi:10.1523/JNEUROSCI.5775-11.2012

7. Martinez TL, Kong L, Wang X, et al. Survival motor neuron protein in motor neurons determines synaptic integrity in spinal muscular atrophy. J Neurosci. Jun 2012;32(25):8703-15. doi:10.1523/JNEUROSCI.0204-12.2012

8. Park GH, Maeno-Hikichi Y, Awano T, Landmesser LT, Monani UR. Reduced survival of motor neuron (SMN) protein in motor neuronal progenitors functions cell autonomously to cause spinal muscular atrophy in model mice expressing the human centromeric (SMN2) gene. $J$ Neurosci. Sep 2010;30(36):12005-19. doi:10.1523/JNEUROSCI.2208-10.2010

9. Lee AJ, Awano T, Park GH, Monani UR. Limited phenotypic effects of selectively augmenting the SMN protein in the neurons of a mouse model of severe spinal muscular atrophy. PLoS One. 2012;7(9):e46353. doi:10.1371/journal.pone.0046353

10. Chen TH. New and Developing Therapies in Spinal Muscular Atrophy: From Genotype to Phenotype to Treatment and Where Do We Stand? Int J Mol Sci. May 07 2020;21(9)doi:10.3390/ijms21093297

11. Pattali R, Mou Y, Li XJ. AAV9 Vector: a Novel modality in gene therapy for spinal muscular atrophy. Gene Ther. 08 2019;26(7-8):287-295. doi:10.1038/s41434-019-0085-4

12. Hinderer C, Katz N, Buza EL, et al. Severe Toxicity in Nonhuman Primates and Piglets Following High-Dose Intravenous Administration of an Adeno-Associated Virus Vector Expressing Human SMN. Hum Gene Ther. 03 2018;29(3):285-298. doi:10.1089/hum.2018.015 13. Van Alstyne M, Tattoli I, Delestrée N, et al. Gain of toxic function by long-term AAV9mediated SMN overexpression in the sensorimotor circuit. Nat Neurosci. Jul 2021;24(7):930940. doi:10.1038/s41593-021-00827-3

14. Sidoryk-Wegrzynowicz M, Wegrzynowicz M, Lee E, Bowman AB, Aschner M. Role of astrocytes in brain function and disease. Toxicol Pathol. Jan 2011;39(1):115-23. doi:10.1177/0192623310385254

15. Chung WS, Allen NJ, Eroglu C. Astrocytes Control Synapse Formation, Function, and Elimination. Cold Spring Harb Perspect Biol. Feb 2015;7(9):a020370. doi:10.1101/cshperspect.a020370

16. Lenz KM, Nelson LH. Microglia and Beyond: Innate Immune Cells As Regulators of Brain Development and Behavioral Function. Front Immunol. 2018;9:698.

doi:10.3389/fimmu.2018.00698

17. Rial D, Lemos C, Pinheiro H, et al. Depression as a Glial-Based Synaptic Dysfunction. Front Cell Neurosci. 2015;9:521. doi:10.3389/fncel.2015.00521 
527

528

529

530

531

532

533

534

535

536

537

538

539

540

541

542

543

544

545

546

547

548

549

550

551

552

553

554

555

556

557

558

559

560

561

562

563

564

565

566

567

568

569

570

571

572

573

574

575

576

577
18. Fontainhas AM, Wang M, Liang KJ, et al. Microglial morphology and dynamic behavior is regulated by ionotropic glutamatergic and GABAergic neurotransmission. PLoS One. Jan 2011;6(1):e15973. doi:10.1371/journal.pone.0015973

19. Schafer DP, Lehrman EK, Stevens B. The "quad-partite" synapse: microglia-synapse interactions in the developing and mature CNS. Glia. Jan 2013;61(1):24-36. doi:10.1002/glia.22389

20. Siracusa R, Fusco R, Cuzzocrea S. Astrocytes: Role and Functions in Brain Pathologies. Front Pharmacol. 2019;10:1114. doi:10.3389/fphar.2019.01114

21. Domeniconi M, Hempstead BL, Chao MV. Pro-NGF secreted by astrocytes promotes motor neuron cell death. Mol Cell Neurosci. Feb 2007;34(2):271-9. doi:10.1016/j.mcn.2006.11.005

22. Matejuk A, Ransohoff RM. Crosstalk Between Astrocytes and Microglia: An Overview. Front Immunol. 2020;11:1416. doi:10.3389/fimmu.2020.01416

23. Fu R, Shen Q, Xu P, Luo JJ, Tang Y. Phagocytosis of microglia in the central nervous system diseases. Mol Neurobiol. Jun 2014;49(3):1422-34. doi:10.1007/s12035-013-8620-6

24. Klawonn AM, Fritz M, Castany S, et al. Microglial activation elicits a negative affective state through prostaglandin-mediated modulation of striatal neurons. Immunity. Feb 2021;54(2):225-234.e6. doi:10.1016/j.immuni.2020.12.016

25. Basu A, Krady JK, O'Malley M, Styren SD, DeKosky ST, Levison SW. The type 1 interleukin-1 receptor is essential for the efficient activation of microglia and the induction of multiple proinflammatory mediators in response to brain injury. J Neurosci. Jul 2002;22(14):6071-82. doi:20026616

26. Brown GC, Neher JJ. Eaten alive! Cell death by primary phagocytosis: 'phagoptosis'.

Trends Biochem Sci. Aug 2012;37(8):325-32. doi:10.1016/j.tibs.2012.05.002

27. Werneburg S, Jung J, Kunjamma RB, et al. Targeted Complement Inhibition at Synapses Prevents Microglial Synaptic Engulfment and Synapse Loss in Demyelinating Disease. Immunity. 01 2020;52(1):167-182.e7. doi:10.1016/j.immuni.2019.12.004

28. Woodruff TM, Costantini KJ, Taylor SM, Noakes PG. Role of complement in motor neuron disease: animal models and therapeutic potential of complement inhibitors. Adv Exp Med Biol. 2008;632:143-58.

29. Heurich B, El Idrissi NB, Donev RM, et al. Complement upregulation and activation on motor neurons and neuromuscular junction in the SOD1 G93A mouse model of familial amyotrophic lateral sclerosis. J Neuroimmunol. Jun 2011;235(1-2):104-9. doi:10.1016/j.jneuroim.2011.03.011

30. McGivern JV, Patitucci TN, Nord JA, Barabas MA, Stucky CL, Ebert AD. Spinal muscular atrophy astrocytes exhibit abnormal calcium regulation and reduced growth factor production. Glia. Sep 2013;61(9):1418-1428. doi:10.1002/glia.22522

31. Patitucci TN, Ebert AD. SMN deficiency does not induce oxidative stress in SMA iPSCderived astrocytes or motor neurons. Hum Mol Genet. Feb 2016;25(3):514-23. doi:10.1093/hmg/ddv489

32. Sison SL, Patitucci TN, Seminary ER, Villalon E, Lorson CL, Ebert AD. Astrocyteproduced miR-146a as a mediator of motor neuron loss in spinal muscular atrophy. Hum Mol Genet. 09 2017;26(17):3409-3420. doi:10.1093/hmg/ddx230

33. Logan S, Arzua T, Canfield SG, et al. Studying Human Neurological Disorders Using Induced Pluripotent Stem Cells: From 2D Monolayer to 3D Organoid and Blood Brain Barrier Models. Compr Physiol. 03 2019;9(2):565-611. doi:10.1002/cphy.c180025

34. Rindt $\mathrm{H}$, Feng Z, Mazzasette $\mathrm{C}$, et al. Astrocytes influence the severity of spinal muscular atrophy. Hum Mol Genet. Jul 2015;24(14):4094-102. doi:10.1093/hmg/ddv148 35. Boopathi E, Hypolite JA, Zderic SA, et al. GATA-6 and NF-KB activate CPI-17 gene transcription and regulate $\mathrm{Ca} 2+$ sensitization of smooth muscle contraction. Mol Cell Biol. Mar 2013;33(5):1085-102. doi:10.1128/MCB.00626-12 
578

579

580

581

582

583

584

585

586

587

588

589

590

591

592

593

594

595

596

597

598

599

600

601

602

603

604

605

606

607

608

609

610

611

612

613

614

615

616

617

618

619

620

621

622

623

624

625

626

36. Yang CW, Chen CL, Chou WC, et al. An Integrative Transcriptomic Analysis for Identifying Novel Target Genes Corresponding to Severity Spectrum in Spinal Muscular Atrophy. PLoS One. 2016;11(6):e0157426. doi:10.1371/journal.pone.0157426

37. Tarabal O, Caraballo-Miralles V, Cardona-Rossinyol A, et al. Mechanisms involved in spinal cord central synapse loss in a mouse model of spinal muscular atrophy. $J$ Neuropathol Exp Neurol. Jun 2014;73(6):519-35. doi:10.1097/NEN.0000000000000074

38. Vukojicic A, Delestrée N, Fletcher EV, et al. The Classical Complement Pathway Mediates Microglia-Dependent Remodeling of Spinal Motor Circuits during Development and in SMA. Cell Rep. 12 2019;29(10):3087-3100.e7. doi:10.1016/j.celrep.2019.11.013

39. Batiuk MY, Martirosyan A, Wahis J, et al. Identification of region-specific astrocyte subtypes at single cell resolution. Nat Commun. 03 2020;11(1):1220. doi:10.1038/s41467-01914198-8

40. Kerstetter AE, Miller RH. Isolation and culture of spinal cord astrocytes. Methods Mol Biol. 2012;814:93-104. doi:10.1007/978-1-61779-452-0_7

41. Tcw J, Wang M, Pimenova AA, et al. An Efficient Platform for Astrocyte Differentiation from Human Induced Pluripotent Stem Cells. Stem Cell Reports. 08 2017;9(2):600-614. doi:10.1016/j.stemcr.2017.06.018

42. de Rus Jacquet A. Preparation and Co-Culture of iPSC-Derived Dopaminergic Neurons and Astrocytes. Curr Protoc Cell Biol. 12 2019;85(1):e98. doi:10.1002/cpcb.98

43. Roybon L, Lamas NJ, Garcia AD, et al. Human stem cell-derived spinal cord astrocytes with defined mature or reactive phenotypes. Cell Rep. Sep 2013;4(5):1035-1048. doi:10.1016/j.celrep.2013.06.021

44. Andersen J, Revah O, Miura Y, et al. Generation of Functional Human 3D Cortico-Motor Assembloids. Cell. 12 2020;183(7):1913-1929.e26. doi:10.1016/j.cell.2020.11.017

45. De Miranda J, Yaddanapudi K, Hornig M, Lipkin WI. Astrocytes recognize intracellular polyinosinic-polycytidylic acid via MDA-5. FASEB J. Apr 2009;23(4):1064-71. doi:10.1096/fj.08121434

46. Soubannier V, Maussion G, Chaineau M, et al. Characterization of human iPSC-derived astrocytes with potential for disease modeling and drug discovery. Neurosci Lett. 0713 2020;731:135028. doi:10.1016/j.neulet.2020.135028

47. Perriot S, Mathias A, Perriard G, et al. Human Induced Pluripotent Stem Cell-Derived Astrocytes Are Differentially Activated by Multiple Sclerosis-Associated Cytokines. Stem Cell Reports. 1113 2018;11(5):1199-1210. doi:10.1016/j.stemcr.2018.09.015

48. Christian F, Smith EL, Carmody RJ. The Regulation of NF-kB Subunits by Phosphorylation. Cells. Mar 2016;5(1)doi:10.3390/cells5010012

49. Baeuerle PA, Baltimore D. I kappa B: a specific inhibitor of the NF-kappa B transcription factor. Science. Oct 1988;242(4878):540-6. doi:10.1126/science.3140380

50. Maury Y, Côme J, Piskorowski RA, et al. Combinatorial analysis of developmental cues efficiently converts human pluripotent stem cells into multiple neuronal subtypes. Nat Biotechnol. Jan 2015;33(1):89-96. doi:10.1038/nbt.3049

51. Stogsdill JA, Eroglu C. The interplay between neurons and glia in synapse development and plasticity. Curr Opin Neurobiol. 02 2017;42:1-8. doi:10.1016/j.conb.2016.09.016 52. Yanuck SF. Microglial Phagocytosis of Neurons: Diminishing Neuronal Loss in Traumatic, Infectious, Inflammatory, and Autoimmune CNS Disorders. Front Psychiatry. 2019;10:712. doi:10.3389/fpsyt.2019.00712

53. Hirsch EC, Hunot S. Neuroinflammation in Parkinson's disease: a target for neuroprotection? Lancet Neurol. Apr 2009;8(4):382-97. doi:10.1016/S1474-4422(09)70062-6

54. Halliday GM, Stevens CH. Glia: initiators and progressors of pathology in Parkinson's disease. Mov Disord. Jan 2011;26(1):6-17. doi:10.1002/mds.23455 
627

628

629

630

631

632

633

634

635

636

637

638

639

640

641

642

643

644

645

646

647

648

649

650

651

652

653

654

655

656

657

658

659

660

661

662

663

664

665

666

667

668

669

670

671

672

673

674

675

676

55. Hong S, Beja-Glasser VF, Nfonoyim BM, et al. Complement and microglia mediate early synapse loss in Alzheimer mouse models. Science. May 2016;352(6286):712-716. doi:10.1126/science.aad8373

56. Kashon ML, Ross GW, O'Callaghan JP, et al. Associations of cortical astrogliosis with cognitive performance and dementia status. J Alzheimers Dis. Dec 2004;6(6):595-604; discussion 673-81. doi:10.3233/jad-2004-6604

57. Haenseler W, Sansom SN, Buchrieser J, et al. A Highly Efficient Human Pluripotent Stem Cell Microglia Model Displays a Neuronal-Co-culture-Specific Expression Profile and Inflammatory Response. Stem Cell Reports. 06 2017;8(6):1727-1742. doi:10.1016/j.stemcr.2017.05.017

58. McQuade A, Coburn M, Tu CH, Hasselmann J, Davtyan H, Blurton-Jones M. Development and validation of a simplified method to generate human microglia from pluripotent stem cells. Mol Neurodegener. 12 2018;13(1):67. doi:10.1186/s13024-018-0297-x

59. Franco-Bocanegra DK, McAuley C, Nicoll JAR, Boche D. Molecular Mechanisms of Microglial Motility: Changes in Ageing and Alzheimer's Disease. Cells. 06 2019;8(6)doi:10.3390/cells8060639

60. Davis BM, Salinas-Navarro M, Cordeiro MF, Moons L, De Groef L. Characterizing microglia activation: a spatial statistics approach to maximize information extraction. Sci Rep. 05 2017;7(1):1576. doi:10.1038/s41598-017-01747-8

61. Sleigh JN, Gillingwater TH, Talbot K. The contribution of mouse models to understanding the pathogenesis of spinal muscular atrophy. Dis Model Mech. Jul 2011;4(4):457-67. doi:10.1242/dmm.007245

62. Rossoll W, Jablonka S, Andreassi C, et al. Smn, the spinal muscular atrophydetermining gene product, modulates axon growth and localization of beta-actin mRNA in growth cones of motoneurons. J Cell Biol. Nov 2003;163(4):801-12. doi:10.1083/jcb.200304128 63. Hua Y, Sahashi K, Rigo F, et al. Peripheral SMN restoration is essential for long-term rescue of a severe spinal muscular atrophy mouse model. Nature. Oct 2011;478(7367):123-6. doi:10.1038/nature 10485

64. Martin JE, Nguyen TT, Grunseich C, et al. Decreased Motor Neuron Support by SMA Astrocytes due to Diminished MCP1 Secretion. J Neurosci. 05 2017;37(21):5309-5318. doi:10.1523/JNEUROSCI.3472-16.2017

65. Cerveró C, Blasco A, Tarabal O, et al. Glial Activation and Central Synapse Loss, but Not Motoneuron Degeneration, Are Prevented by the Sigma-1 Receptor Agonist PRE-084 in the Smn2B/- Mouse Model of Spinal Muscular Atrophy. J Neuropathol Exp Neurol. 07 2018;77(7):577-597. doi:10.1093/jnen/nly033

66. Ling KK, Lin MY, Zingg B, Feng Z, Ko CP. Synaptic defects in the spinal and neuromuscular circuitry in a mouse model of spinal muscular atrophy. PLoS One. Nov 2010;5(11):e15457. doi:10.1371/journal.pone.0015457

67. Morel L, Men Y, Chiang MSR, et al. Intracortical astrocyte subpopulations defined by astrocyte reporter Mice in the adult brain. Glia. 01 2019;67(1):171-181. doi:10.1002/glia.23545 68. Hochstim C, Deneen B, Lukaszewicz A, Zhou Q, Anderson DJ. Identification of positionally distinct astrocyte subtypes whose identities are specified by a homeodomain code. Cell. May 02 2008;133(3):510-22. doi:10.1016/j.cell.2008.02.046

69. Tsai HH, Li H, Fuentealba LC, et al. Regional astrocyte allocation regulates CNS synaptogenesis and repair. Science. Jul 20 2012;337(6092):358-62.

doi:10.1126/science.1222381

70. Sofroniew MV. Astrocyte failure as a cause of CNS dysfunction. Mol Psychiatry. May 2000;5(3):230-2.

71. Neumann M, Naumann M. Beyond IkappaBs: alternative regulation of NF-kappaB activity. FASEB J. Sep 2007;21(11):2642-54. doi:10.1096/fj.06-7615rev 
677

678

679

680

681

682

683

684

685

686

687

688

689

690

691

692

693

694

695

696

697

698

699

700

701

702

703

704

705

706

707

708

709

710

711

712

713

714

715

716

717

718

719

720

721

722

723

724

725

726

72. Wu X, Chen PS, Dallas S, et al. Histone deacetylase inhibitors up-regulate astrocyte GDNF and BDNF gene transcription and protect dopaminergic neurons. Int $J$ Neuropsychopharmacol. Dec 2008;11(8):1123-34. doi:10.1017/S1461145708009024

73. Kumar P, Godbole NM, Chaturvedi CP, et al. Mechanisms involved in epigenetic downregulation of Gfap under maternal hypothyroidism. Biochem Biophys Res Commun. 07 2018;502(3):375-381. doi:10.1016/j.bbrc.2018.05.173

74. Litvinchuk A, Wan YW, Swartzlander DB, et al. Complement C3aR Inactivation Attenuates Tau Pathology and Reverses an Immune Network Deregulated in Tauopathy Models and Alzheimer's Disease. Neuron. 12 2018;100(6):1337-1353.e5. doi:10.1016/j.neuron.2018.10.031

75. Harder JM, Williams PA, Braine CE, et al. Complement peptide C3a receptor 1 promotes optic nerve degeneration in DBA/2J mice. J Neuroinflammation. Nov 2020;17(1):336. doi:10.1186/s12974-020-02011-z

76. Zhang LY, Pan J, Mamtilahun M, et al. Microglia exacerbate white matter injury via complement C3/C3aR pathway after hypoperfusion. Theranostics. 2020;10(1):74-90. doi:10.7150/thno.35841

77. Neher JJ, Neniskyte U, Zhao JW, Bal-Price A, Tolkovsky AM, Brown GC. Inhibition of microglial phagocytosis is sufficient to prevent inflammatory neuronal death. $J$ Immunol. Apr 15 2011;186(8):4973-83. doi:10.4049/jimmunol.1003600

78. Neniskyte U, Neher JJ, Brown GC. Neuronal death induced by nanomolar amyloid $\beta$ is mediated by primary phagocytosis of neurons by microglia. J Biol Chem. Nov 18 2011;286(46):39904-13. doi:10.1074/jbc.M111.267583

79. Fricker M, Neher JJ, Zhao JW, Théry C, Tolkovsky AM, Brown GC. MFG-E8 mediates primary phagocytosis of viable neurons during neuroinflammation. J Neurosci. Feb 22 2012;32(8):2657-66. doi:10.1523/JNEUROSCI.4837-11.2012

80. Ricklin D, Reis ES, Mastellos DC, Gros P, Lambris JD. Complement component C3 The "Swiss Army Knife" of innate immunity and host defense. Immunol Rev. 11 2016;274(1):3358. doi:10.1111/imr.12500

81. Carpanini SM, Torvell M, Morgan BP. Therapeutic Inhibition of the Complement System in Diseases of the Central Nervous System. Front Immunol. 2019;10:362. doi:10.3389/fimmu.2019.00362

82. Peterson SL, Nguyen HX, Mendez OA, Anderson AJ. Complement Protein C3 Suppresses Axon Growth and Promotes Neuron Loss. Sci Rep. 10 2017;7(1):12904. doi:10.1038/s41598-017-11410-x

83. Park JS, Kam TI, Lee S, et al. Blocking microglial activation of reactive astrocytes is neuroprotective in models of Alzheimer's disease. Acta Neuropathol Commun. 0426 2021;9(1):78. doi:10.1186/s40478-021-01180-z

84. Savage JC, St-Pierre MK, Carrier M, et al. Microglial physiological properties and interactions with synapses are altered at presymptomatic stages in a mouse model of Huntington's disease pathology. J Neuroinflammation. Apr 02 2020;17(1):98. doi:10.1186/s12974-020-01782-9

85. Yun SP, Kam TI, Panicker N, et al. Block of A1 astrocyte conversion by microglia is neuroprotective in models of Parkinson's disease. Nat Med. 07 2018;24(7):931-938. doi:10.1038/s41591-018-0051-5

86. Liddelow SA, Guttenplan KA, Clarke LE, et al. Neurotoxic reactive astrocytes are induced by activated microglia. Nature. 0126 2017;541(7638):481-487.

doi:10.1038/nature21029

87. Mejzini R, Flynn LL, Pitout IL, Fletcher S, Wilton SD, Akkari PA. ALS Genetics, Mechanisms, and Therapeutics: Where Are We Now? Front Neurosci. 2019;13:1310. doi:10.3389/fnins.2019.01310 
727 88. Frakes AE, Ferraiuolo L, Haidet-Phillips AM, et al. Microglia induce motor neuron death

728 via the classical NF-kB pathway in amyotrophic lateral sclerosis. Neuron. Mar 2014;81(5):1009-

729 1023. doi:10.1016/j.neuron.2014.01.013

730 89. Franklin H, Clarke BE, Patani R. Astrocytes and microglia in neurodegenerative

731 diseases: Lessons from human in vitro models. Prog Neurobiol. May 2021;200:101973.

732 doi:10.1016/j.pneurobio.2020.101973 
bioRxiv preprint doi: https://doi.org/10.1101/2021.10.11.463972; this version posted October 12, 2021. The copyright holder for this preprint (which was not certified by peer review) is the author/funder, who has granted bioRxiv a license to display the preprint in perpetuity. It is made available under aCC-BY-NC-ND 4.0 International license.
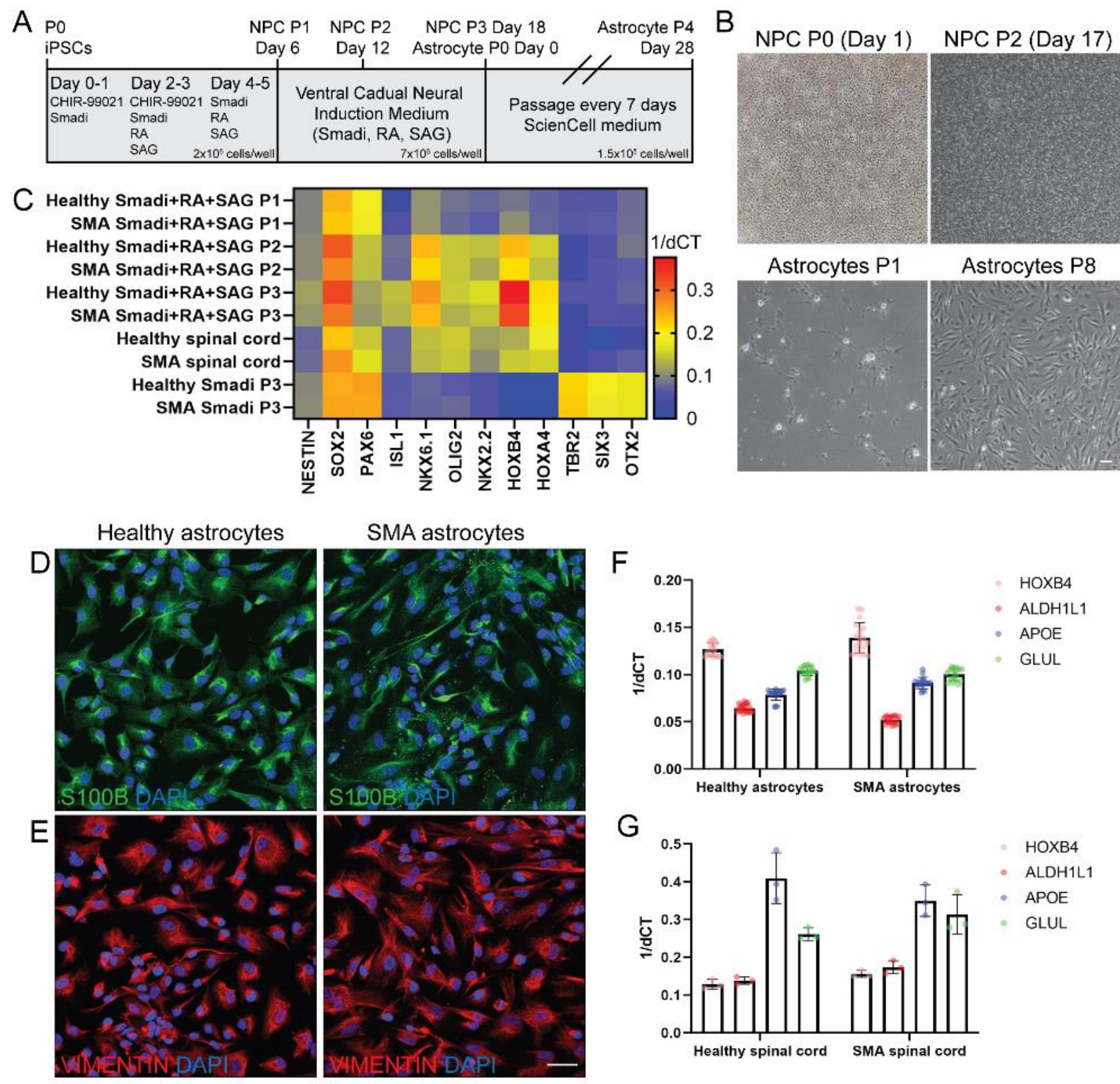

\section{G}
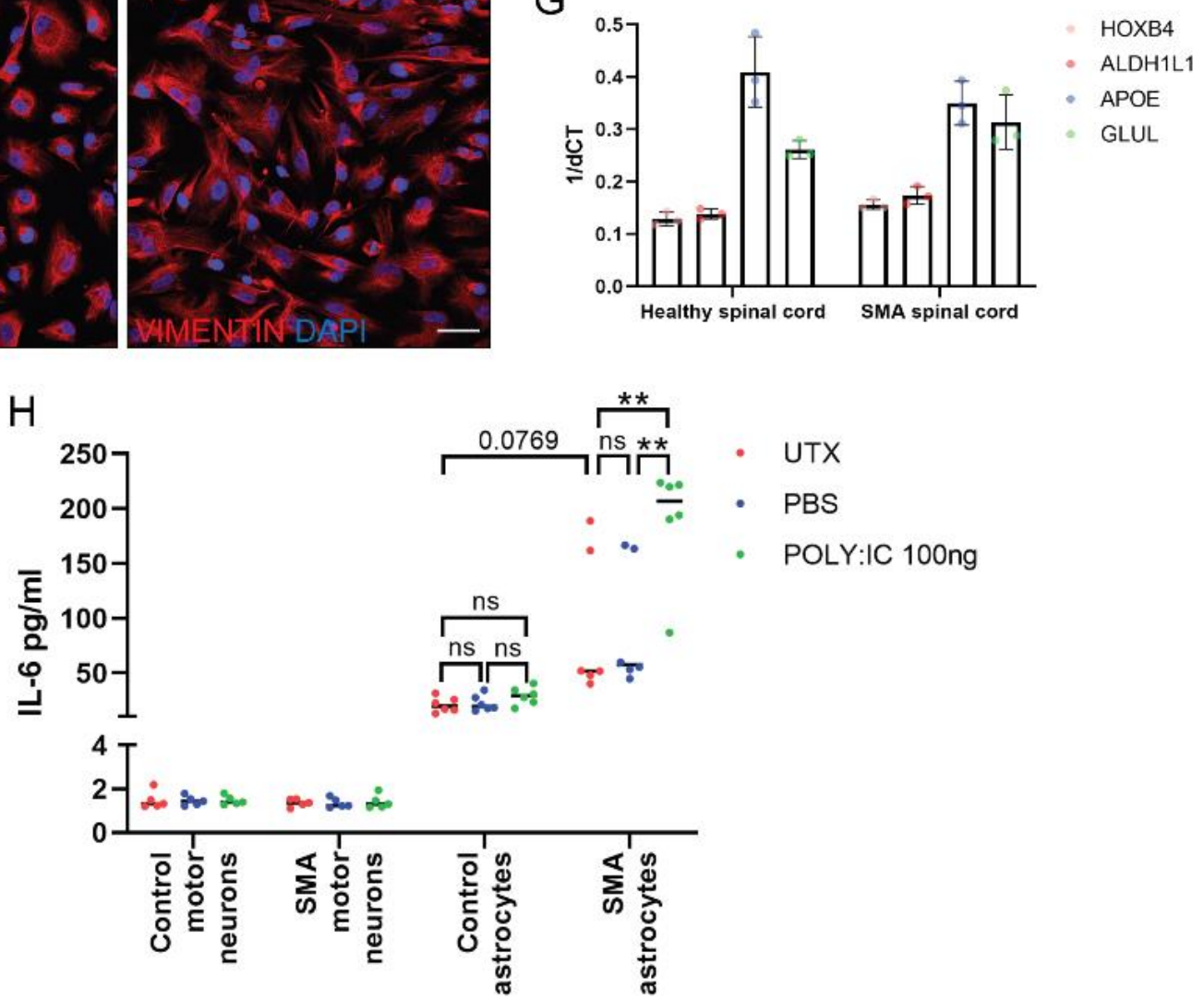
736 Figure 1. Spinal cord astrocyte differentiation of induced pluripotent stem cells (iPSCs). (A)

737 Schematic of neural progenitor cell (NPC) and astrocyte differentiation protocols from iPSCs. NPCs are

738 patterned and cultured for Day 18 before beginning astrocyte differentiation. Astrocytes beyond P4 (Day

739 28) are considered fully differentiated. (B) Representative brightfield images of NPC and astrocyte

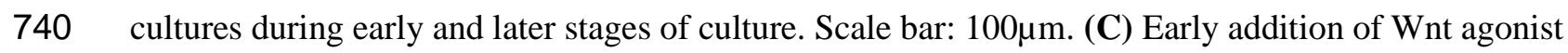

741 (CHIR 99021), SMAD inhibition (SB431542 and LDN-193189), retinoic acid (RA) and hedgehog

742 smoothened agonist (SAG) during NPC differentiation (P1-3) increases the expression of general NPC-

743 associated genes (NESTIN, SOX2, PAX6) and spinal cord ventral caudal-associated genes (ISL1,

744 NKX6.1, OLIG2, HOXB4 and HOXA4). Post-mortem spinal cord and ventral-caudal patterned NPC (P2

745 onwards) samples show comparable gene expression profiles, whereas Smadi only treated NPCs show

746 increased forebrain transcription factor gene expression (TBR2, SIX3, OTX2). Markers characteristic of

747 astrocyte identity, including S100B (D) VIMENTIN (E), ALDH1L1, APOE, GLUL (F), and spinal cord

748 ventral caudal transcription factor HOXB4 (D) are readily detected in astrocytes differentiated from NPC

749 cultures and in post-mortem spinal cord samples $(\mathbf{G})$. Scale bar: 50 $\mu$ m. (H) Significantly elevated levels

750 of interleukin-6 (IL-6) are detected in conditioned media from stimulated (POLY:IC treated) SMA

751 astrocytes, compared to untreated (UTX) and vehicle treated (PBS) SMA astrocytes, and equivalent

752 healthy astrocyte samples. iPSC-derived motor neurons served as a negative control for this assay. 2-way

753 ANOVA, Tukey's multiple comparisons test. **pvalue $<0.005$. 
bioRxiv preprint doi: https://doi.org/10.1101/2021.10.11.463972; this version posted October 12, 2021. The copyright holder for this preprint (which was not certified by peer review) is the author/funder, who has granted bioRxiv a license to display the preprint in perpetuity. It is made available under aCC-BY-NC-ND 4.0 International license.

A

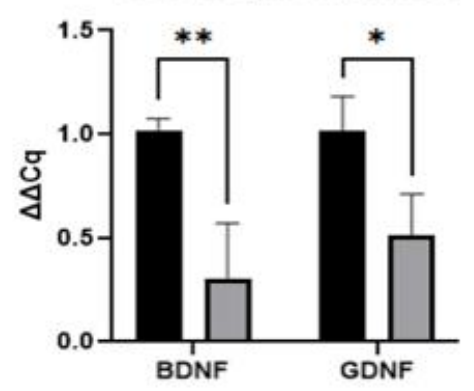

B
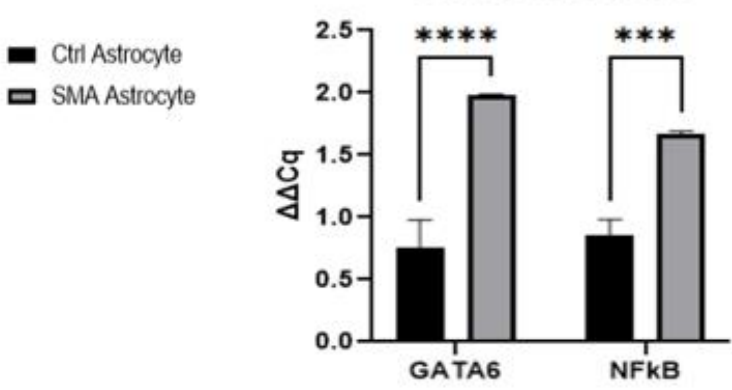

- Ctri Astrocyte

口 SMA Astrocyte
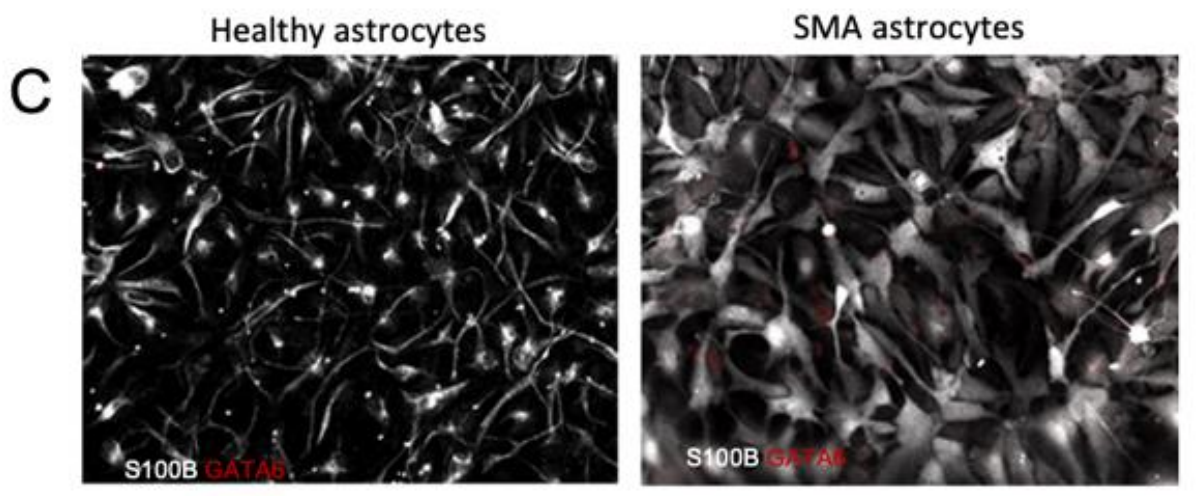

E

Protein Expression

D
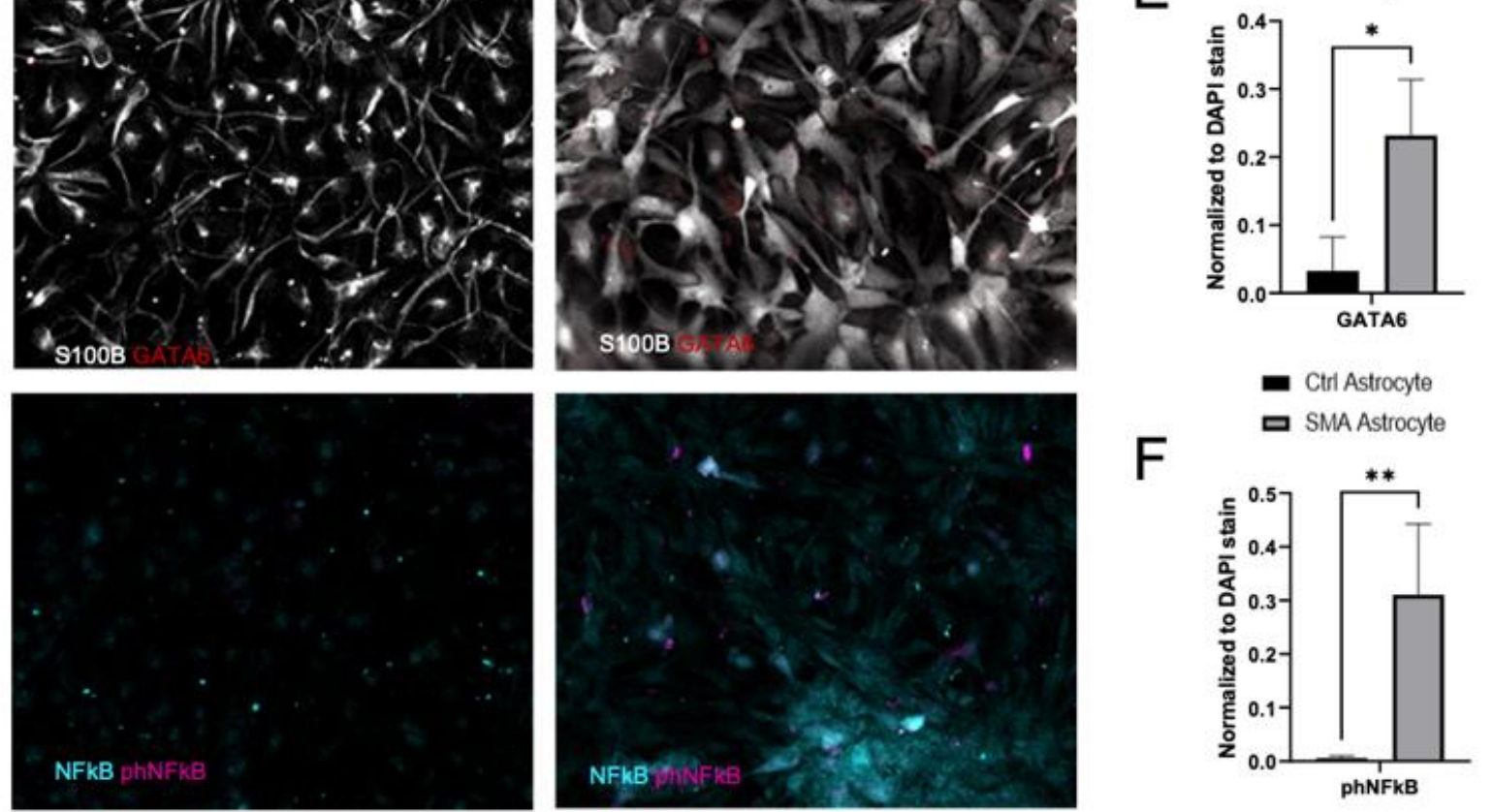

$\mathrm{F}$

$$
\text { - Ctri Astrocyte }
$$$$
\text { 口 SMA Astrocyte }
$$

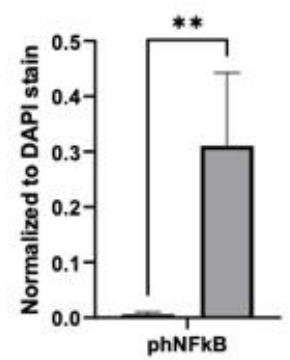

G

Inflammatory Ligands

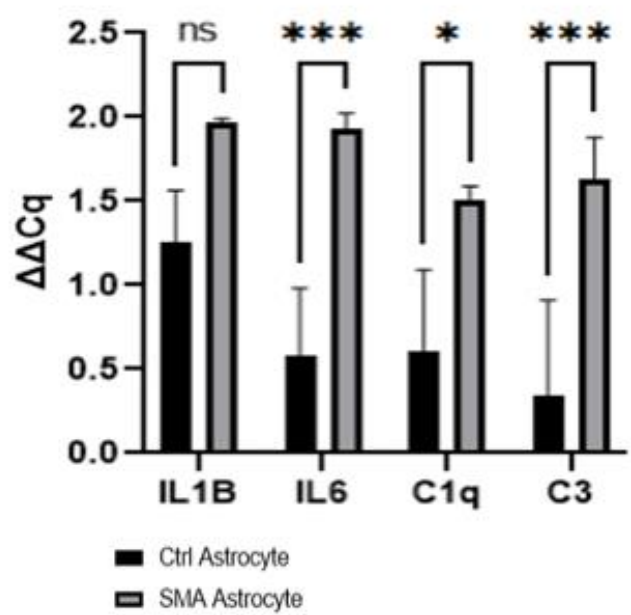

$\mathrm{H}$ Astrocyte Markers

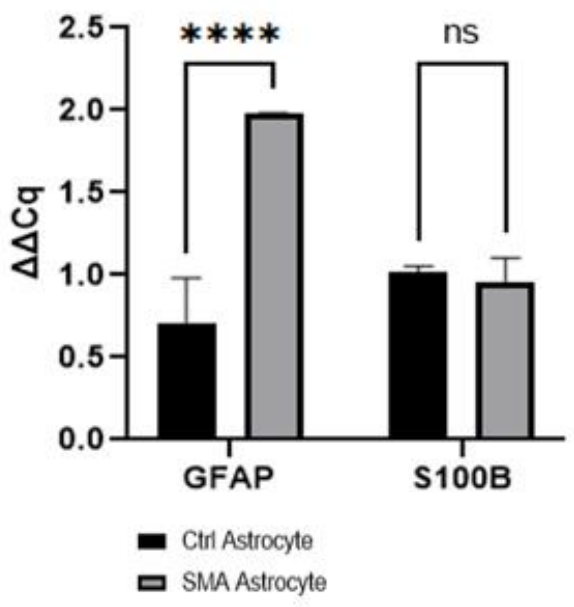


756 Figure 2. GATA6 and nuclear NFKB upregulation in SMA astrocytes. (A) Decreased neurotrophic

757 support through BDNF, GDNF and (B) increased GATA6, NFKB transcript production confirmed in

758 SMA astrocytes compared to healthy controls. (C) Representative images of GATA6 and (D) phNFкB

759 protein expression in SMA and Ctrl Astrocytes by immunocytochemistry (E, F quantification). (G)

760 Upregulation of transcripts for pro-inflammatory cytokines and complement factors (IL1 $\beta$, IL6, C1q, C3)

761 and $\mathbf{( H )}$ activation marker GFAP in SMA astrocytes compared to Ctrl, with no changes in general

762 astrocyte marker S100 $\beta$ expression. 1-way ANOVA, Tukey's multiple comparisons test. *pvalue $<0.05$,

$763 * *<0.005, * * *<0.0005, * * * *<0.0001$. 
bioRxiv preprint doi: https://doi.org/10.1101/2021.10.11.463972; this version posted October 12, 2021. The copyright holder for this preprint (which was not certified by peer review) is the author/funder, who has granted bioRxiv a license to display the preprint in perpetuity. It is made available under aCC-BY-NC-ND 4.0 International license.

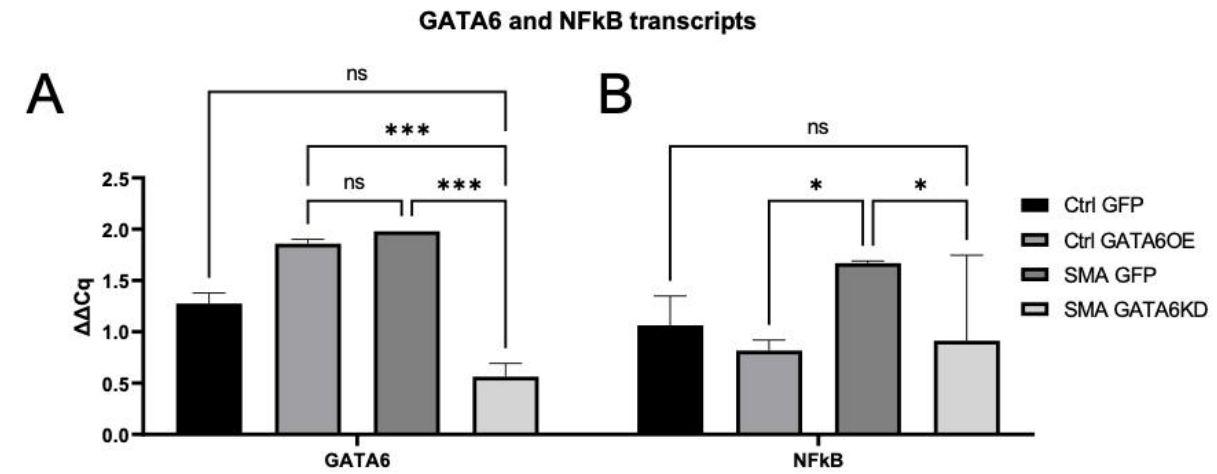

Ctrl GATA6OE astrocytes

C
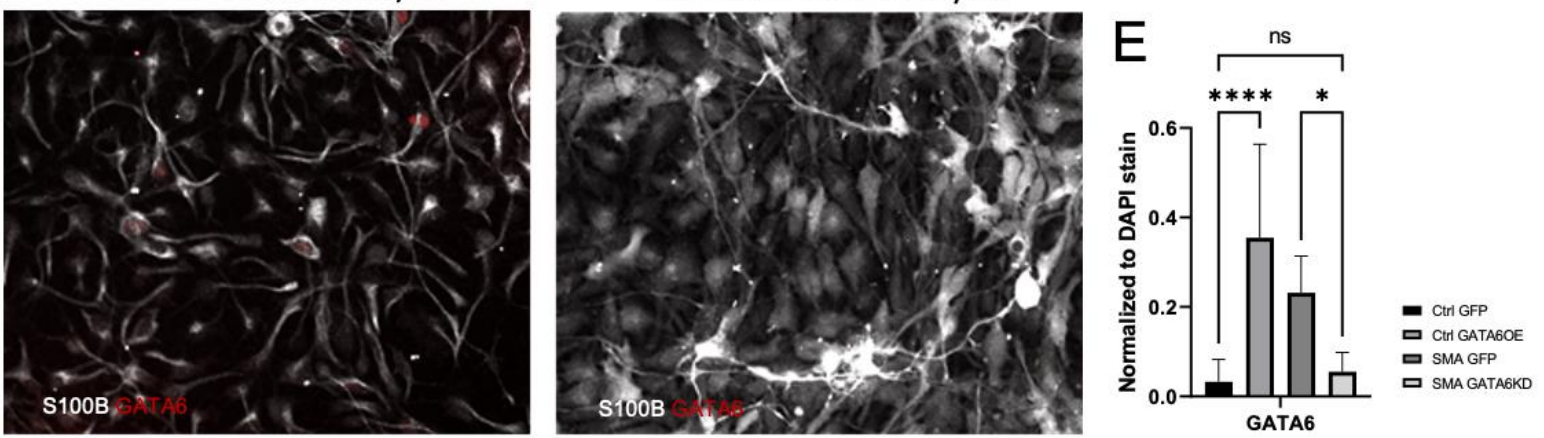

D
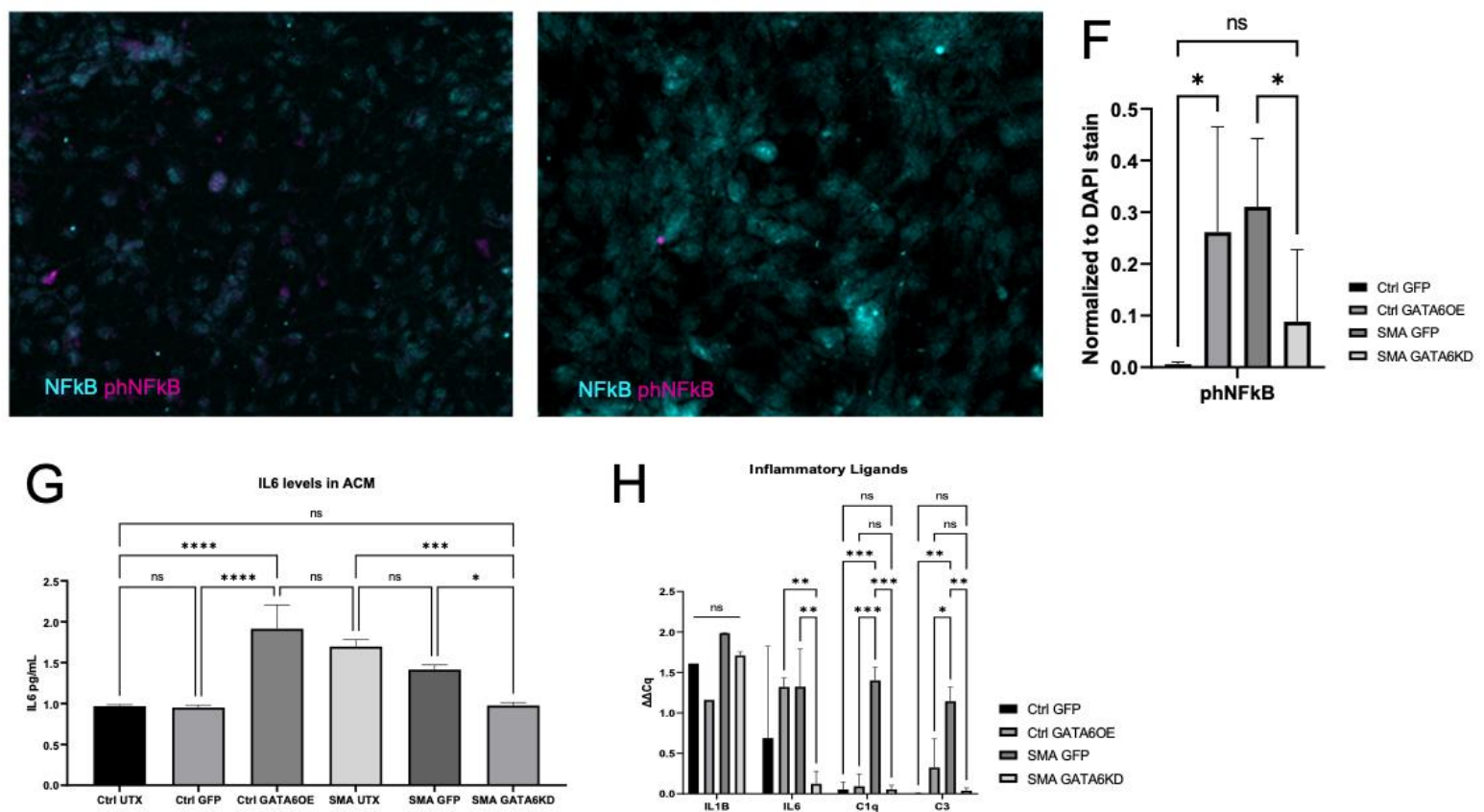

$\mathrm{H}$
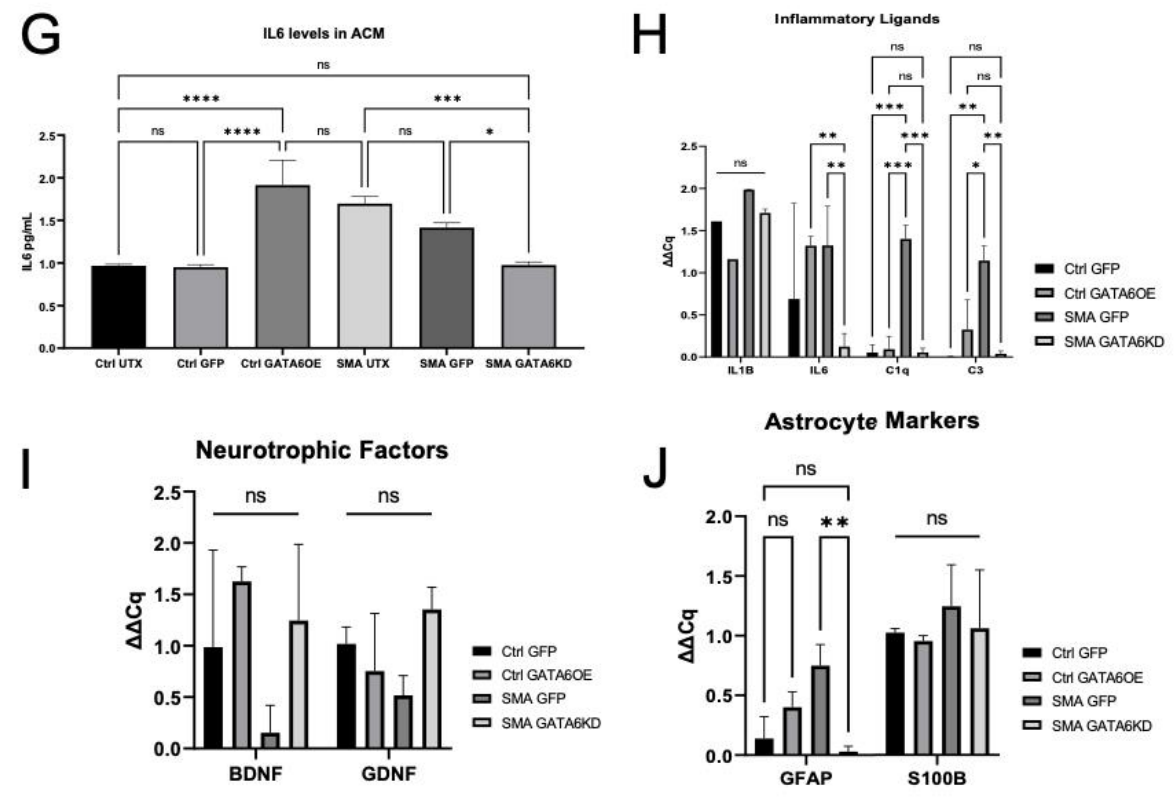
766 Figure 3. Changes in inflammatory and neurotrophic gene expression after astrocyte GATA6

767 manipulation. (A) Confirmation of GATA6 transcript reduction (SMA) or overexpression (Ctrl) after

768 exposure to GFP control, GATA6 expressing, or GATA6KD lentivirus and (B) associated change in

769 NFkB transcript production. (C, D) Confirmation of GATA6 and phNFkB protein expression changes

770 after lentiviral manipulation assessed by ICC (E, F quantification). (G) Secreted IL6 protein levels

771 confirm transcript changes after GATA6 manipulation. (H) Significant decrease in IL6, C1q, and C3

772 transcripts after GATA6KD in SMA astrocytes, trends for increases after GATA6OE in Ctrl astrocytes.

773 (I) No significant changes in neurotrophic support after GATA6 manipulation. (J) Significant decrease in

774 reactive GFAP transcripts, but importantly not in characteristic marker S100ß. 2-way ANOVA, Tukey’s

775 multiple comparisons test for qPCR; 1-way ANOVA, Tukey's multiple comparisons test for ICC,

$776 \quad *$ pvalue $<0.05, * *<0.005, * * *<0.0005, * * * *<0.0001$. 
bioRxiv preprint doi: https://doi.org/10.1101/2021.10.11.463972; this version posted October 12, 2021. The copyright holder for this preprint (which was not certified by peer review) is the author/funder, who has granted bioRxiv a license to display the preprint in perpetuity. It is made available under aCC-BY-NC-ND 4.0 International license.
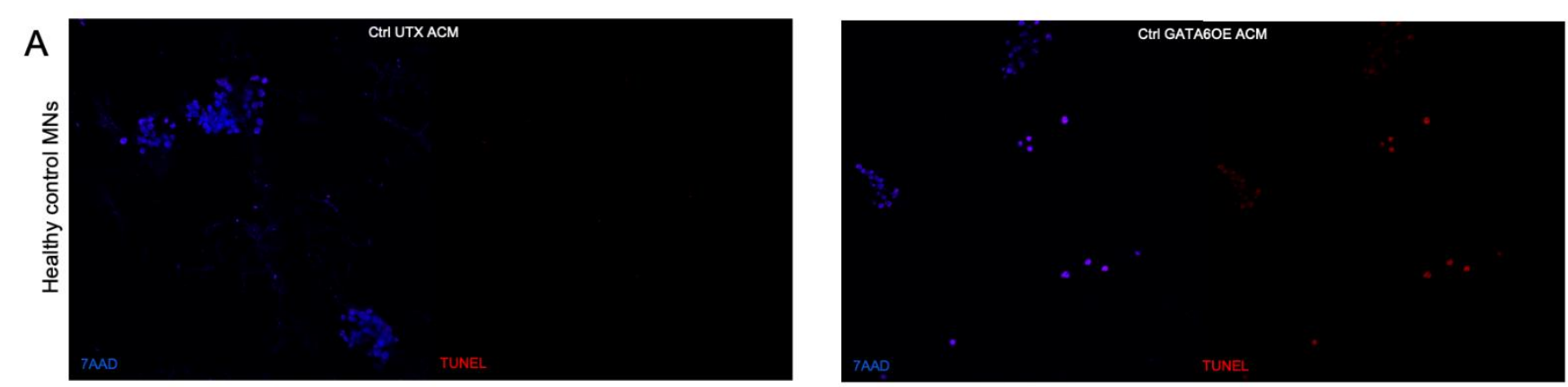

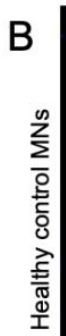

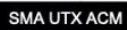

SMA GATAGKD ACM

\section{Ctrl MNs}

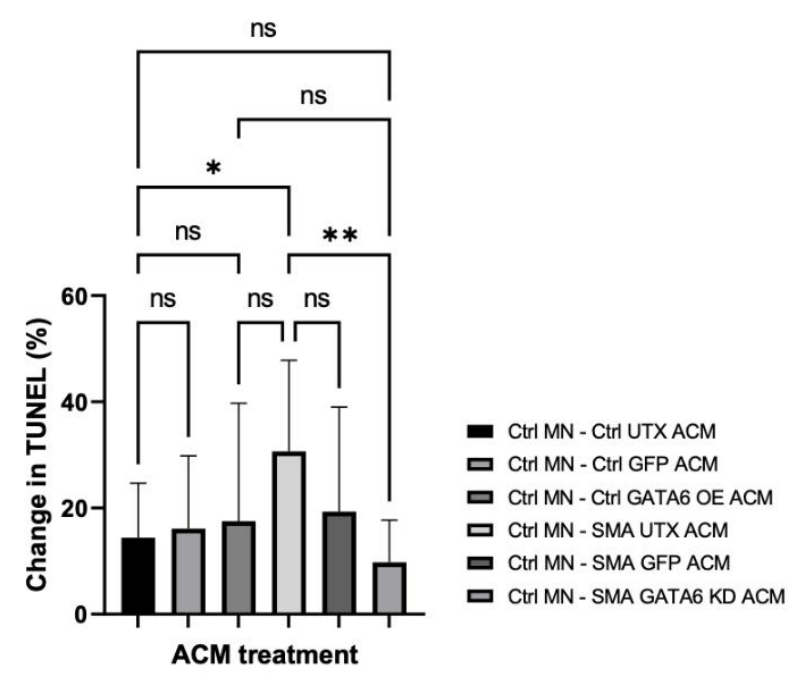


bioRxiv preprint doi: https://doi.org/10.1101/2021.10.11.463972; this version posted October 12, 2021. The copyright holder for this preprint (which was not certified by peer review) is the author/funder, who has granted bioRxiv a license to display the preprint in perpetuity. It is made available under aCC-BY-NC-ND 4.0 International license.
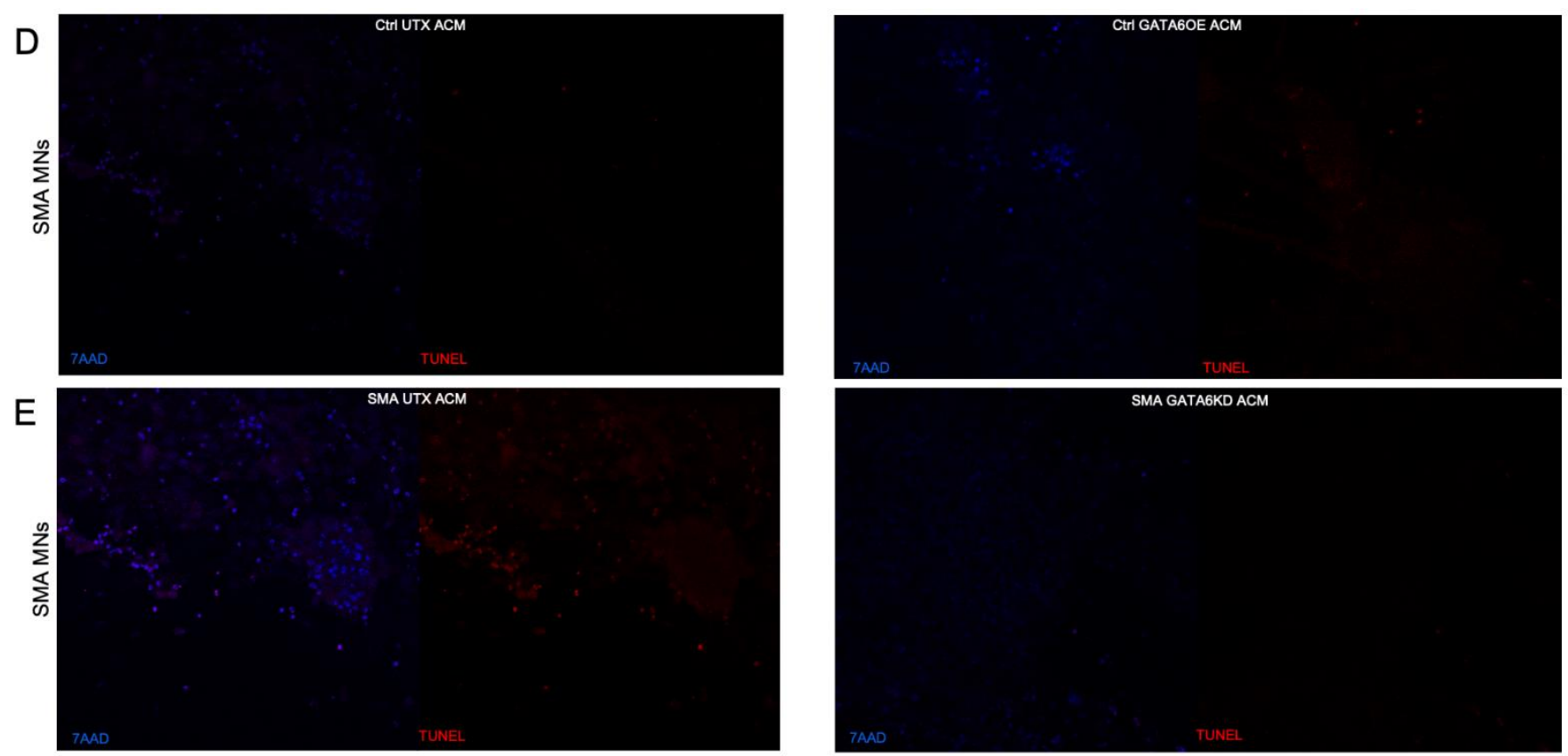

$\mathrm{F}$

SMA MNs

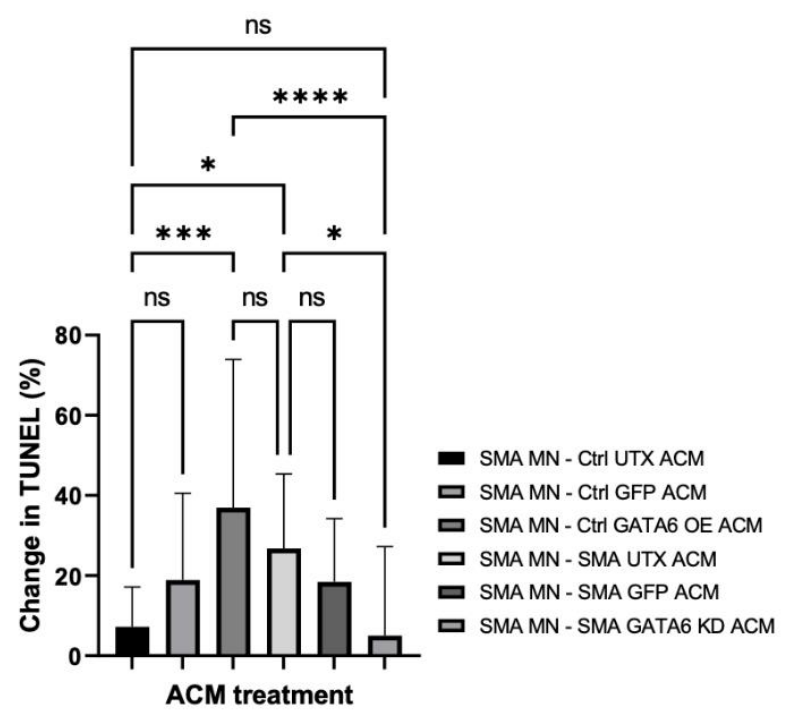


779 Figure 4. Spinal motor neuron survival after astrocyte GATA6 manipulation. Conditioned media

780 from Ctrl untreated (UTX) or GATA6OE astrocytes applied onto Ctrl (A) and SMA (D) spinal motor

781 neurons (MN). Media from SMA UTX or GATA6KD astrocytes also applied onto Ctrl (B) and SMA (E)

782 MNs. Cell death was assessed with a TUNEL kit. TUNEL stain was normalized to live cell nuclear

783 7AAD stain and represented as percent change from the first WT UTX ACM replicate for Ctrl and SMA

784 MNs (C, F). In Ctrl and SMA MNs, SMA UTX was significantly more toxic than Ctrl ACM, an effect

785 which GATA6KD ameliorated. GATA6OE was significantly more toxic than Ctrl UTX only when

786 applied onto SMA MNs. No differences in cell death were found between UTX and GFP conditions. 1-

787 way ANOVA, Tukey's multiple comparisons test. *pvalue $<0.05, * *<0.005, * * *<0.0005, * * * *<0.0001$. 
bioRxiv preprint doi: https://doi.org/10.1101/2021.10.11.463972; this version posted October 12, 2021. The copyright holder for this preprint (which was not certified by peer review) is the author/funder, who has granted bioRxiv a license to display the preprint in perpetuity. It is made available under aCC-BY-NC-ND 4.0 International license.

A

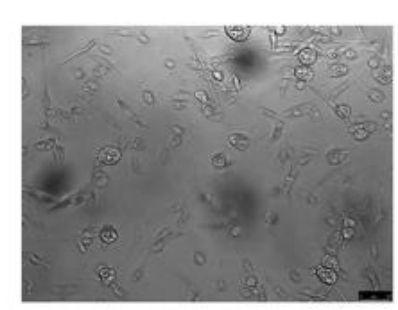

C

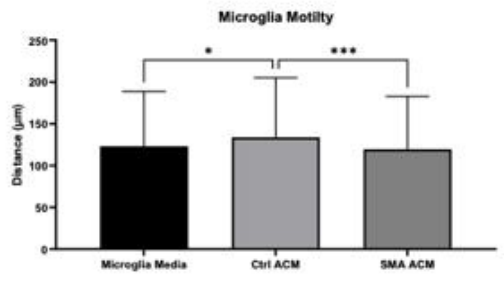

$\mathrm{E}$
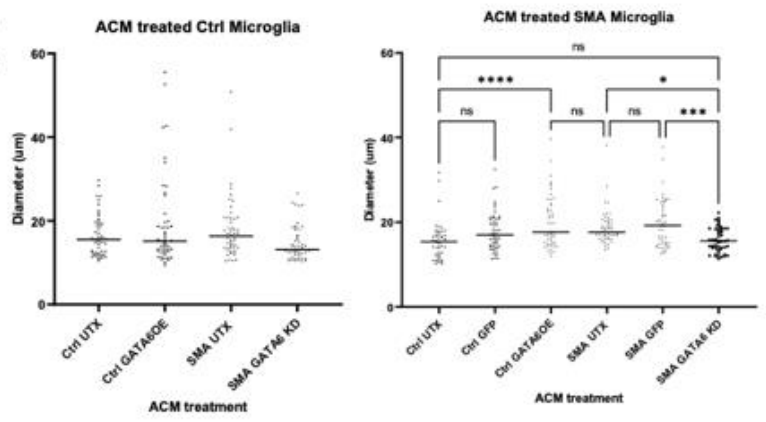

G

MCM treated SMA Astrocytes

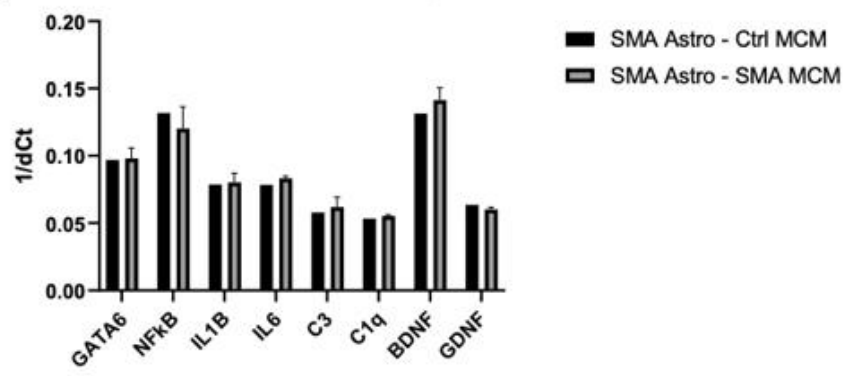

B Microglia Markers
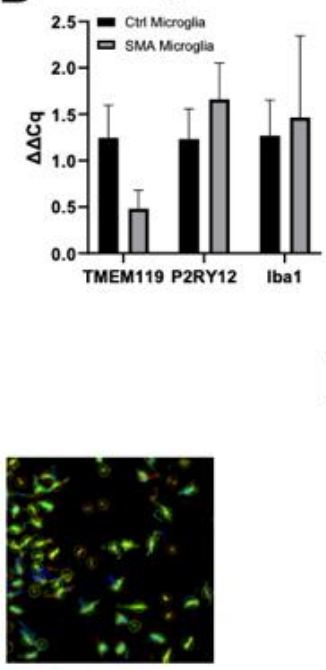

ACM treatment

- SMA Astro - CtrI MCM

口 SMA Astro - SMA MCM

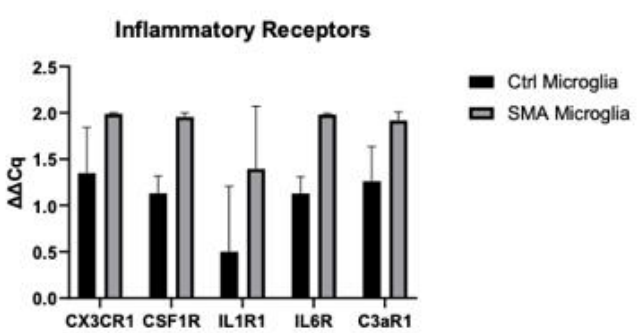

D Microglia Phagocytosis

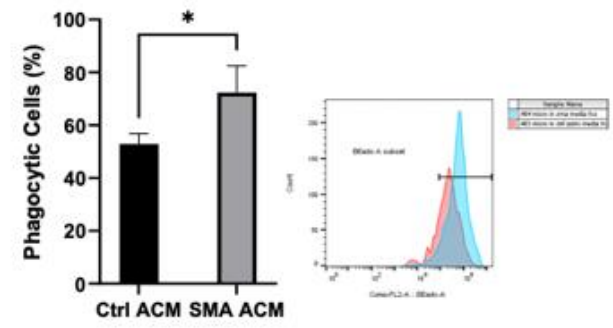

$\mathrm{F}$

Phagocytic SMA Microglia

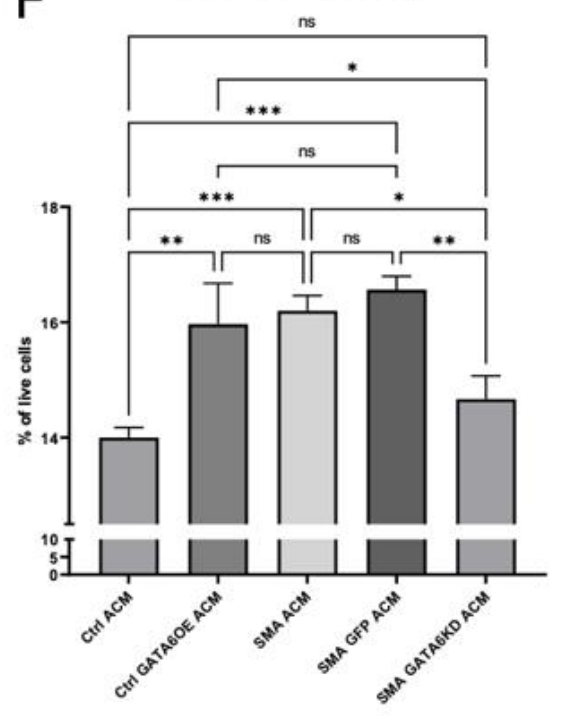


Figure 5. Microglial activation after exposure to astrocyte conditioned media (ACM). iPSC-derived

790 microglia from Ctrl and SMA lines express microglia-specific markers TMEM119, P2RY12, and Iba1 (A

791 representative image, B). (B) SMA Microglia show trends for upregulated transcripts for inflammatory

792 receptors including CX3CR1, CSF1R, IL1R1, IL6R, and C3aR1 (ns). (C) SMA microglia exposed to Ctrl

793 ACM show increased motility compared to SMA ACM in live image tracking (left - representative image,

794 right - quantification). (D) SMA Microglia show increased phagocytosis of FluoSpheres after SMA ACM

795 treatment compared to Ctrl ACM. (E, left) No changes in phenotype (soma diameter) after Ctrl Microglia

796 treated with GATA6 manipulated ACM. (E, right) A significant increase in soma size of SMA Microglia

797 treated with GATA6OE compared to Ctrl UTX was found, as well as a significant decrease after

798 GATA6KD compared to SMA UTX. (F) Significantly decreased phagocytosis of FluoSpheres when

799 SMA Microglia treated with GATA6LD ACM compared to SMA UTX and SMA GFP ACM. (G) No

800 changes found in any transcripts after SMA Astrocytes treated with microglia conditioned media. 1-way

801 ANOVA, Tukey's multiple comparisons test. *pvalue $<0.05, * *<0.005, * * *<0.0005, * * * *<0.0001$. 